CRYSTALLOGRAPHIC COMMUNICATIONS

ISSN 2056-9890

Received 21 March 2015

Accepted 10 June 2015

Edited by M. Weil, Vienna University of Technology, Austria

Keywords: crystal structure; redetermination; system $\mathrm{Pd}-\mathrm{Sn}$; defect variant of the $\mathrm{AlB}_{2}$ structure type

CCDC reference: 1406124

Supporting information: this article has

supporting information at journals.iucr.org/e

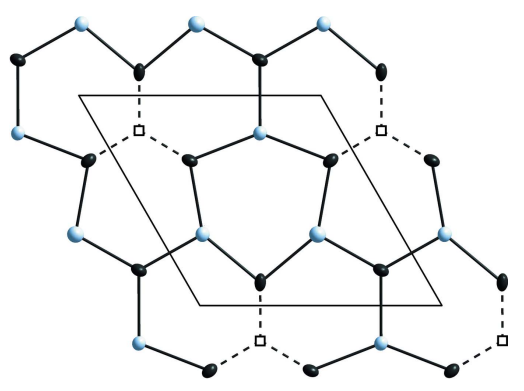

OPEN $\odot$ ACCESS

\section{' $\mathrm{Pd}_{20} \mathrm{Sn}_{13}$ ' revisited: crystal structure of $\mathrm{Pd}_{6.69} \mathrm{Sn}_{4.31}$}

\author{
Wilhelm Klein, Hanpeng Jin, Viktor Hlukhyy and Thomas F. Fässler*
}

Technische Universität München, Department of Chemistry, Lichtenbergstrasse 4, 85747 Garching, Germany. *Correspondence e-mail: thomas.faessler@Irz.tu-muenchen.de

The crystal structure of the title compound was previously reported with composition ' $\mathrm{Pd}_{20} \mathrm{Sn}_{13}$ ' [Sarah et al. (1981). Z. Metallkd, 72, 517-520]. For the original structure model, as determined from powder X-ray data, atomic coordinates from the isostructural compound $\mathrm{Ni}_{13} \mathrm{Ga}_{3} \mathrm{Ge}_{6}$ were transferred. The present structure determination, resulting in a composition $\mathrm{Pd}_{6.69} \mathrm{Sn}_{4.31}$, is based on single crystal X-ray data and includes anisotropic displacement parameters for all atoms as well as standard uncertainties for the atomic coordinates, leading to higher precision and accuracy for the structure model. Single crystals of the title compound were obtained via a solid-state reaction route, starting from the elements. The crystal structure can be derived from the $\mathrm{AlB}_{2}$ type of structure after removing one eighth of the atoms at the boron positions and shifting adjacent atoms in the same layer in the direction of the voids. One atomic site is partially occupied by both elements with a Pd:Sn ratio of 0.38 (3):0.62 (3). One Sn and three Pd atoms are located on special positions with site symmetry 2. (Wyckoff letter $3 a$ and $3 b$ ).

\section{Chemical context}

In the context of investigations of the binary system $\mathrm{Pd}-\mathrm{Sn}$, Nowotny et al. (1946) observed a phase with approximate composition $\mathrm{Pd}_{3} \mathrm{Sn}_{2}$, which was later addressed as ' $\mathrm{Pd}_{20} \mathrm{Sn}_{13}$ ' (Sarah et al., 1981). According to powder XRD measurements, this compound was found to be isotypical to $\mathrm{Ni}_{13} \mathrm{Ga}_{3} \mathrm{Ge}_{6}$ (Nover \& Schubert, 1981). Up to now, no further detailed structure examination has been published. In the course of our experiments, aiming at ternary Zintl phases containing tetrel elements (Hlukhyy et al., 2012), single crystals of the title compound have been obtained in significant amounts and were subjected to a closer structural investigation.

\section{Structural commentary}

The crystal structure of the title compound can be described as a defect variant of the $\mathrm{AlB}_{2}$ structure type, where $1 / 8$ of the boron atoms are missing. The symmetry reduction from $P 6 / \mathrm{mmm}$ to $P 3_{2} 21$ with respect to $\mathrm{AlB}_{2}$ results in 13 different crystallographic positions for the $\mathrm{Pd}$ and $\mathrm{Sn}$ atoms instead of only two, and a more complicated stacking of atomic planes including six differently packed layers for each of the former two, as shown in Fig. 1. The remaining atomic sites of the B atoms in $\mathrm{AlB}_{2}$ are now substituted by seven independent atoms (Pd6, Pd7, Pd8, Sn2, Sn3, Sn4, and Sn5), the 'Al' layers are substituted alternatingly by $\mathrm{Sn} 1, \mathrm{Pd} 3, \mathrm{Pd} 5$, (layers 'Al1', 'Al3', 'Al5' in Fig. 1), and by Pd1, Pd2, and Pd4 ('Al2', 'Al4', 'Al6'), respectively.

The layered character of the $\mathrm{Pd}_{6.69} \mathrm{Sn}_{4.31}$ structure is much less pronounced than in the parent $\mathrm{AlB}_{2}$ type of structure, as indicated by the mixed substitution of both the $\mathrm{Al}$ and $\mathrm{B}$ sites 


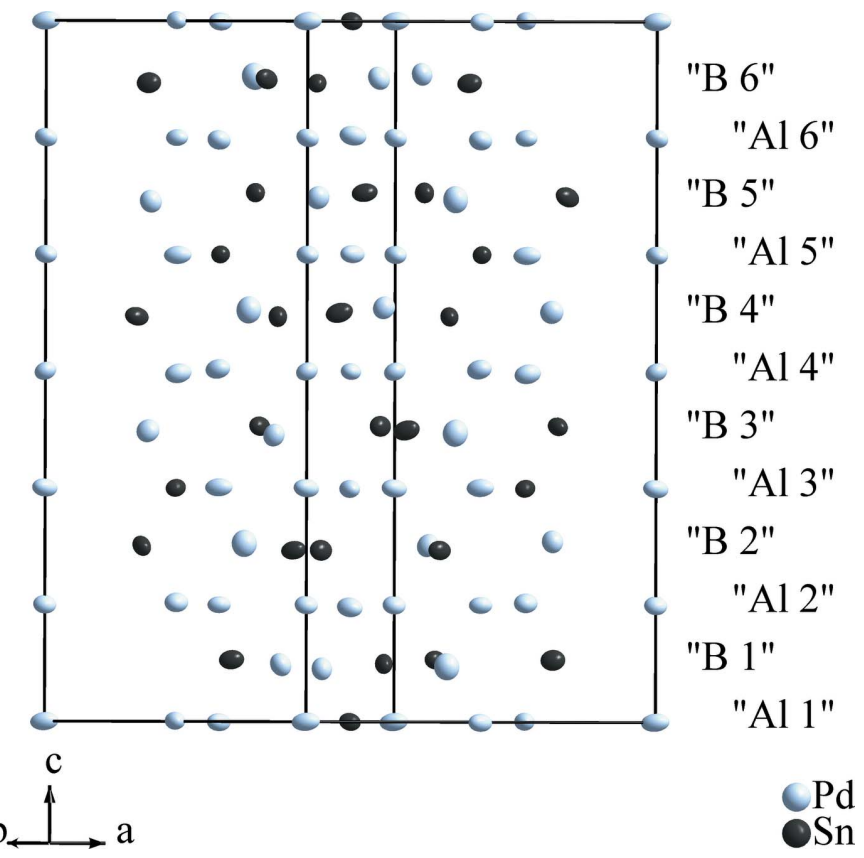

Figure 1

The crystal structure of $\mathrm{Pd}_{6.69} \mathrm{Sn}_{4.31}$, emphasizing the relationship to the $\mathrm{AlB}_{2}$ structure type. The ' $\mathrm{Al} n$ ' layers represent planes which are occupied by $\mathrm{Al}$ atoms in $\mathrm{AlB}_{2}$, the ' $\mathrm{B} n$ ' layers those with $\mathrm{B}$ atoms, respectively. Anisotropic displacement ellipsoids are drawn at the $90 \%$ probability level.

of the $\mathrm{AlB}_{2}$ type by $\mathrm{Pd}$ as well as by $\mathrm{Sn}$ atoms, respectively. Accordingly, there are similar, in average slightly shorter interatomic distances within the planes (2.6407(19) 2.755 (2) ̊) than between them [2.7259 (18)-3.309 (2) ̊]. Nevertheless, the layers are clearly distinguishable and only marginally puckered. The distorted honeycomb lattice is obvious if the voids in the ' $\mathrm{B}$ ' layer are considered (Fig. 2). The distortion results from a shift of the neighbouring Sn atoms

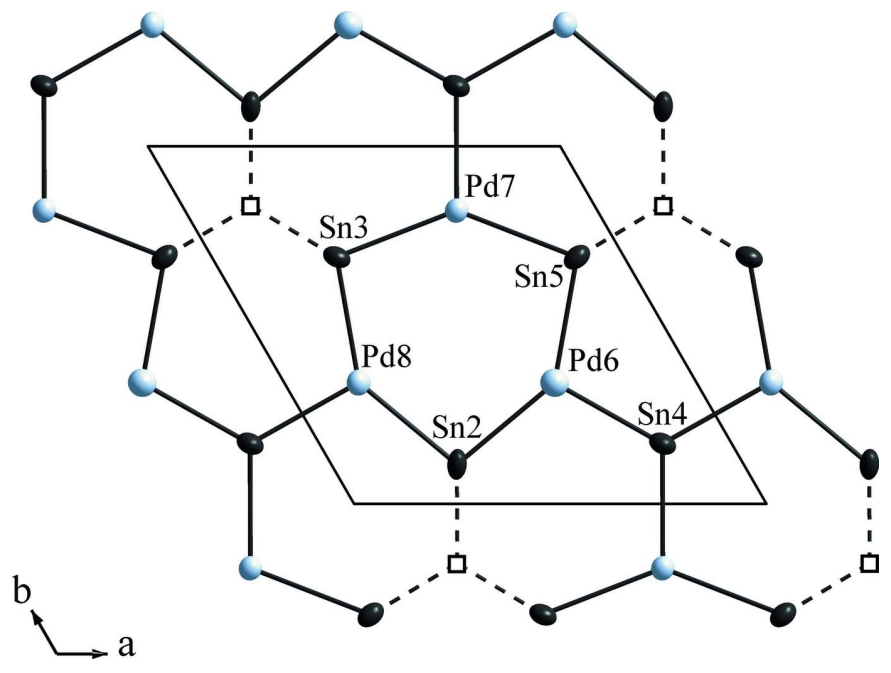

Figure 2

The 'B1' layer (see Fig. 1) in $\mathrm{Pd}_{6.69} \mathrm{Sn}_{4.31}$. To illustrate the relationship to the $\mathrm{AlB}_{2}$ structure type, the voids are drawn as empty squares and are connected to the neighbouring Sn atoms by dashed lines. Anisotropic displacement ellipsoids are drawn at the $90 \%$ probability level. a)

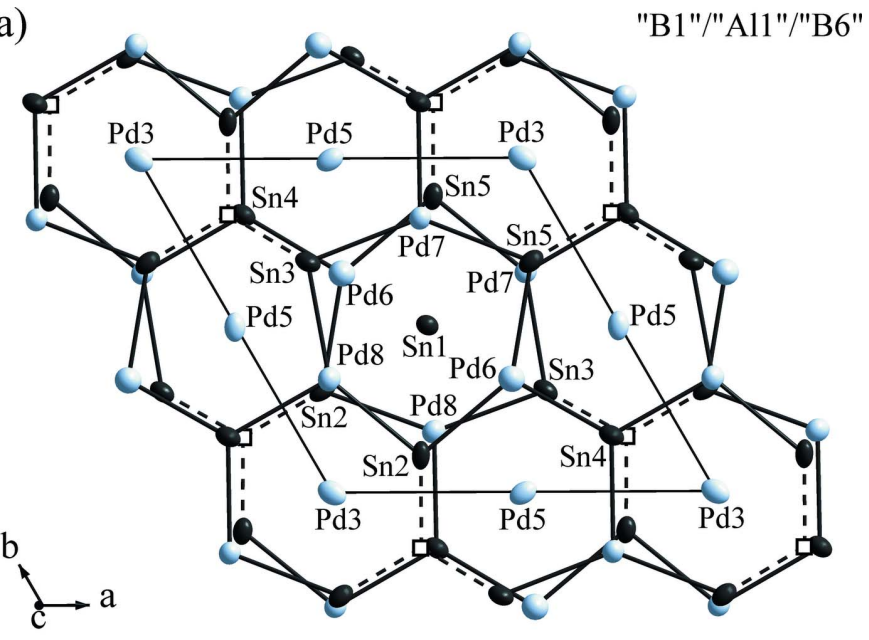

b)

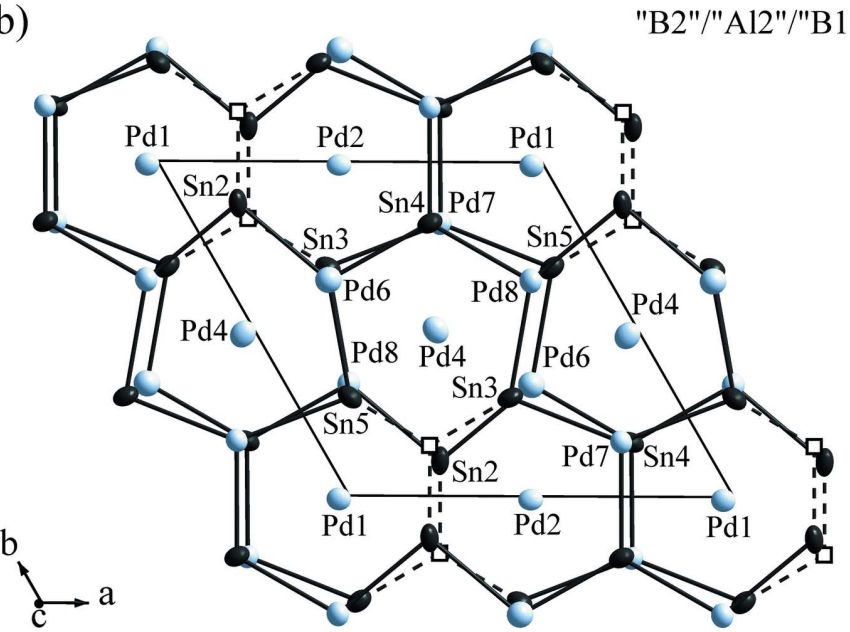

Figure 3

Sections of the crystal structure of $\mathrm{Pd}_{6.69} \mathrm{Sn}_{4.31}$, with $a$ ) layers 'B1'-'Al1''B6' and $b$ ) layers 'B2'-'A12'-'B1'. The voids are drawn as empty squares and are connected to the neighbouring $\mathrm{Sn}$ atoms by dashed lines. Shown are the surroundings of the 'B' layer atoms with zero (Sn1), one (Pd4) and two voids (Pd1, Pd2, Pd3, Pd5). Anisotropic displacement ellipsoids are drawn at the $90 \%$ probability level.

within the boron layer ( $\mathrm{Sn} 2, \mathrm{Sn} 3$ and $\mathrm{Sn} 5)$ in the direction of the voids.

For $\mathrm{Sn} 1$ a partial occupation by $\mathrm{Pd}(\mathrm{Pd} 9)$ was found. A full occupation of the (Sn1/Pd9) site (Fig. $3 a)$ by the element $\mathrm{Sn}$ would result in the composition $\mathrm{Pd}_{13} \mathrm{Sn}_{9}$ as suggested by the isostructural compound $\mathrm{Ni}_{13} \mathrm{Ga}_{3} \mathrm{Ge}_{6}$. However, the occupancy of this position (in contrast to all other $\mathrm{Pd}$ and $\mathrm{Sn}$ sites) deviates significantly from $100 \%$ if only Sn (refined to $96 \%$ ) or $\mathrm{Pd}$ (refined to $107 \%$ ) is considered. It has to be noticed that this site is the only one in both kinds of 'Al' layers that is not close to a void in the ' $\mathrm{B}$ ' layers (Fig. 3). Consequently, the coordination number $(\mathrm{CN})$ of the $(\mathrm{Sn} 1 / \mathrm{Pd} 9)$ site is 14 , which is higher than that of all other $\mathrm{Sn}(\mathrm{CN}=10)$ and $\mathrm{Pd}$ atoms $(\mathrm{CN}=$ 11-13) in $\mathrm{Pd}_{6.69} \mathrm{Sn}_{4.31}$.

In the previous structure report of ' $\mathrm{Pd}_{20} \mathrm{Sn}_{13}$ ' by Sarah et al. (1981), the atomic parameters were adopted from the isostructural compound $\mathrm{Ni}_{13} \mathrm{Ga}_{3} \mathrm{Ge}_{6}$, and the occupation of 
Table 1

Experimental details.

\begin{tabular}{|c|c|}
\hline \multicolumn{2}{|l|}{ Crystal data } \\
\hline Chemical formula & $\mathrm{Pd}_{6.69} \mathrm{Sn}_{4.31}$ \\
\hline$M_{\mathrm{r}}$ & 1223.37 \\
\hline Crystal system, space group & Trigonal, $P 3_{2} 21$ \\
\hline Temperature $(\mathrm{K})$ & 150 \\
\hline$a, c(\AA)$ & $8.77574(17), 16.9004(4)$ \\
\hline$V\left(\AA^{3}\right)$ & $1127.18(5)$ \\
\hline$Z$ & 6 \\
\hline Radiation type & Mo $K \alpha$ \\
\hline$\mu\left(\mathrm{mm}^{-1}\right)$ & 29.54 \\
\hline Crystal size (mm) & $0.16 \times 0.1 \times 0.08$ \\
\hline \multicolumn{2}{|l|}{ Data collection } \\
\hline Diffractometer & Oxford Xcalibur 3 \\
\hline Absorption correction & $\begin{array}{l}\text { Multi-scan (CrysAlis RED; Oxford } \\
\quad \text { Diffraction, 2009) }\end{array}$ \\
\hline$T_{\min }, T_{\max }$ & $0.408,1.000$ \\
\hline $\begin{array}{l}\text { No. of measured, independent and } \\
\text { observed }[I>2 \sigma(I)] \text { reflections }\end{array}$ & $20534,2682,2001$ \\
\hline$R_{\text {int }}$ & 0.041 \\
\hline$(\sin \theta / \lambda)_{\max }\left(\AA^{-1}\right)$ & 0.762 \\
\hline \multicolumn{2}{|l|}{ Refinement } \\
\hline$R\left[F^{2}>2 \sigma\left(F^{2}\right)\right], w R\left(F^{2}\right), S$ & $0.028,0.076,1.08$ \\
\hline No. of reflections & 2682 \\
\hline No. of parameters & 104 \\
\hline$\Delta \rho_{\max }, \Delta \rho_{\min }\left(\mathrm{e} \AA^{-3}\right)$ & $2.66,-2.52$ \\
\hline Absolute structure & $\begin{array}{l}\text { Flack } x \text { determined using } 715 \\
\text { quotients } \\
{\left[\left(I^{+}\right)-\left(I^{-}\right)\right] /\left[\left(I^{+}\right)+\left(I^{-}\right)\right]} \\
\quad \text { (Parsons } \text { et al., } 2013)\end{array}$ \\
\hline Absolute structure parameter & $-0.2(2)$ \\
\hline
\end{tabular}

Computer programs: CrysAlis CCD and CrysAlis RED (Oxford Diffraction, 2009), SHELXS97 (Sheldrick, 2008), SHELXL2014 (Sheldrick, 2015) and DIAMOND (Brandenburg, 2012).

one atomic site was fixed for $\mathrm{Sn}: \mathrm{Pd}$ as $2 / 3: 1 / 3$. The composition ' $\mathrm{Pd}_{20} \mathrm{Sn}_{13}$ ' was obviously chosen in order to get the indices as integers, however, in consequence $Z=2$. Our structure refinement suggests a more precise composition $\mathrm{Pd}_{20.06(5)} \mathrm{Sn}_{12.94(5)}$. With a crystallographically more appropriate number of formula units, viz. $Z=6$ (indicating the asymmetric unit), the composition then refined to $\mathrm{Pd}_{6.69(2)} \mathrm{Sn}_{4.31(2)}$.

\section{Synthesis and crystallization}

Single crystals of the title compound were obtained from experiments aiming at a ternary alloy in the chemical system $\mathrm{K}-\mathrm{Pd}-\mathrm{Sn}$, with similar conditions as reported by Hlukhyy et al. (2012). $23.4 \mathrm{mg} \mathrm{K}$ (99.9\%, Riedel de Haën), $71 \mathrm{mg}$ Sn (99.999\%, ChemPur), and $20.6 \mathrm{mg}$ of PdSn, prefabricated by arc melting of the elements, were filled into a niobium crucible, which was sealed, placed in a silica glass tube, annealed under vacuum for $20 \mathrm{~h}$ at $1273 \mathrm{~K}$ and subsequently for $72 \mathrm{~h}$ at $873 \mathrm{~K}$, and finally quenched with liquid nitrogen. As a by-product, $\mathrm{K}_{4} \mathrm{Sn}_{4}$ (Hewaidy et al., 1964) was found.

\section{Refinement}

Crystal data, data collection and structure refinement details are summarized in Table 1 . In contrast to the previously reported structure model, which was described in $P 3_{1} 21$ (based on powder X-ray data; Sarah et al., 1981), the crystal under investigation adopts the inverted structure, as indicated by the refined Flack parameter (Flack, 1983; Parsons et al., 2013). Therefore space group $P 3_{2} 21$ was chosen for the current refinement. It should be noted that the value and the corresponding standard uncertainty for the Flack parameter are rather high. However, the cause for this behaviour remains unclear. For the $\mathrm{Sn} 1$ site a partial occupation by $\mathrm{Pd}(\mathrm{Pd} 9)$ was found, with a refined occupation of 62 (3)\% Sn and 38 (3)\% $\mathrm{Pd}$. All atoms were refined with anisotropic displacement parameters. The remaining maximum and minimum electron densities are located $2.08 \AA$ from $\mathrm{Sn} 2$ and $0.46 \AA$ from $\mathrm{Pd} 8$, respectively.

\section{Acknowledgements}

This work was supported by the German Research Foundation (DFG) and the Technische Universität München within the funding programme Open Access Publishing.

\section{References}

Brandenburg, K. (2012). DIAMOND. Crystal Impact GbR, Bonn, Germany.

Flack, H. D. (1983). Acta Cryst. A39, 876-881.

Hewaidy, I. F., Busmann, E. \& Klemm, W. (1964). Z. Anorg. Allg. Chem. 328, 283-293.

Hlukhyy, V., He, H., Jantke, L. A. \& Fässler, T. F. (2012). Chem. Eur. J. 18, 12000-120007.

Nover, G. \& Schubert, K. (1981). Z. Metallkd, 72, 26-29.

Nowotny, H., Schubert, K. \& Dettinger, U. (1946). Z. Metallkd, 37, 137.

Oxford Diffraction (2009). CrysAlis CCD and CrysAlis RED. Agilent Technologies UK Ltd, Yarnton, Oxfordshire, England.

Parsons, S., Flack, H. D. \& Wagner, T. (2013). Acta Cryst. B69, 249259.

Sarah, N., Alasafi, K. \& Schubert, K. (1981). Z. Metallkd, 72, 517-520.

Sheldrick, G. M. (2008). Acta Cryst. A64, 112-122.

Sheldrick, G. M. (2015). Acta Cryst. C71, 3-8. 


\section{supporting information}

Acta Cryst. (2015). E71, 807-809 [doi:10.1107/S2056989015011366]

\section{' $P d_{20} S_{13}$ ' revisited: crystal structure of $\mathbf{P d}_{6.69} \mathrm{Sn}_{4.31}$}

\section{Wilhelm Klein, Hanpeng Jin, Viktor Hlukhyy and Thomas F. Fässler}

\section{Computing details}

Data collection: CrysAlis CCD (Oxford Diffraction, 2009); cell refinement: CrysAlis RED (Oxford Diffraction, 2009); data reduction: CrysAlis RED (Oxford Diffraction, 2009); program(s) used to solve structure: SHELXS97 (Sheldrick, 2008); program(s) used to refine structure: SHELXL2014 (Sheldrick, 2015); molecular graphics: DIAMOND (Brandenburg, 2012); software used to prepare material for publication: SHELXL2014 (Sheldrick, 2015).

\section{Heptapalladium tetratin}

Crystal data

$\mathrm{Pd}_{6.69} \mathrm{Sn}_{4.31}$

$M_{r}=1223.37$

Trigonal, $P 3_{2} 21$

$a=8.77574$ (17) $\AA$

$c=16.9004$ (4) $\AA$

$V=1127.18(5) \AA^{3}$

$Z=6$

$F(000)=3139$

Data collection

Oxford Xcalibur 3

diffractometer

Radiation source: Enhance (Mo) X-ray Source Graphite monochromator

Detector resolution: 16.0238 pixels $\mathrm{mm}^{-1}$

$\omega$ and $\pi$ scans

Absorption correction: multi-scan

(CrysAlis RED; Oxford Diffraction, 2009)

$T_{\min }=0.408, T_{\max }=1.000$

Refinement

Refinement on $F^{2}$

Least-squares matrix: full

$R\left[F^{2}>2 \sigma\left(F^{2}\right)\right]=0.028$

$w R\left(F^{2}\right)=0.076$

$S=1.08$

2682 reflections

104 parameters

0 restraints

$w=1 /\left[\sigma^{2}\left(F_{\mathrm{o}}^{2}\right)+(0.0364 P)^{2}+1.004 P\right]$

where $P=\left(F_{\mathrm{o}}^{2}+2 F_{\mathrm{c}}^{2}\right) / 3$
$D_{\mathrm{x}}=10.813 \mathrm{Mg} \mathrm{m}^{-3}$

Mo $K \alpha$ radiation, $\lambda=0.71073 \AA$

Cell parameters from 8247 reflections

$\theta=2.9-32.7^{\circ}$

$\mu=29.54 \mathrm{~mm}^{-1}$

$T=150 \mathrm{~K}$

Fragment, black

$0.16 \times 0.1 \times 0.08 \mathrm{~mm}$

20534 measured reflections

2682 independent reflections

2001 reflections with $I>2 \sigma(I)$

$R_{\text {int }}=0.041$

$\theta_{\text {max }}=32.8^{\circ}, \theta_{\text {min }}=2.9^{\circ}$

$h=-13 \rightarrow 8$

$k=-12 \rightarrow 12$

$l=-25 \rightarrow 25$

$(\Delta / \sigma)_{\max }<0.001$

$\Delta \rho_{\max }=2.66 \mathrm{e}^{-3}$

$\Delta \rho_{\min }=-2.52$ e $\AA^{-3}$

Extinction correction: SHELXL2014 (Sheldrick, 2015), $\mathrm{Fc}^{*}=\mathrm{kFc}\left[1+0.001 \mathrm{xFc}^{2} \lambda^{3} / \sin (2 \theta)\right]^{-1 / 4}$

Extinction coefficient: 0.00066 (4)

Absolute structure: Flack $x$ determined using 715 quotients $[(\mathrm{I}+)-(\mathrm{I}-)] /[(\mathrm{I}+)+(\mathrm{I}-)]$ (Parsons et al., 2013)

Absolute structure parameter: -0.2 (2) 


\section{Special details}

Geometry. All e.s.d.'s (except the e.s.d. in the dihedral angle between two 1.s. planes) are estimated using the full covariance matrix. The cell e.s.d.'s are taken into account individually in the estimation of e.s.d.'s in distances, angles and torsion angles; correlations between e.s.d.'s in cell parameters are only used when they are defined by crystal symmetry. An approximate (isotropic) treatment of cell e.s.d.'s is used for estimating e.s.d.'s involving 1.s. planes.

Fractional atomic coordinates and isotropic or equivalent isotropic displacement parameters $\left(\AA^{2}\right)$

\begin{tabular}{llllll}
\hline & $x$ & $y$ & $z$ & $U_{\text {iso }} * U_{\text {eq }}$ & Occ. $(<1)$ \\
\hline Pd1 & $-0.0002(5)$ & 0.0000 & 0.1667 & $0.0083(2)$ & \\
Pd2 & $0.4990(2)$ & 0.0000 & 0.1667 & $0.00772(19)$ & \\
Pd3 & $-0.0010(5)$ & $-0.0010(5)$ & 0.0000 & $0.0100(2)$ & \\
Pd4 & $0.5041(2)$ & $0.5072(3)$ & $0.16345(4)$ & $0.01021(19)$ & \\
Pd5 & $0.4963(2)$ & $-0.0035(5)$ & $-0.00092(4)$ & $0.00921(17)$ & \\
Pd6 & $0.6556(3)$ & $0.3385(3)$ & $0.07906(6)$ & $0.0124(2)$ & \\
Pd7 & $0.6590(3)$ & $0.82039(16)$ & $0.07559(5)$ & $0.00882(17)$ & \\
Pd8 & $0.18110(16)$ & $0.3394(3)$ & $0.08142(5)$ & $0.00947(16)$ & \\
Pd9 & $0.4997(2)$ & $0.4997(2)$ & 0.0000 & $0.0071(3)$ & $0.37(4)$ \\
Sn1 & $0.4997(2)$ & $0.4997(2)$ & 0.0000 & $0.0071(3)$ & $0.63(4)$ \\
Sn2 & $0.3048(2)$ & $0.11037(11)$ & $0.08285(5)$ & $0.00898(19)$ & \\
Sn3 & $0.3030(3)$ & $0.6900(3)$ & $0.08831(5)$ & $0.0083(2)$ & \\
Sn4 & $0.83212(15)$ & $0.16829(14)$ & $0.08857(4)$ & $0.00861(14)$ & \\
Sn5 & $0.88531(10)$ & $0.6896(2)$ & $0.08862(5)$ & $0.0084(2)$ & \\
& & & & & \\
\hline
\end{tabular}

Atomic displacement parameters $\left(\AA^{2}\right)$

\begin{tabular}{lllllll}
\hline & $U^{11}$ & $U^{22}$ & $U^{33}$ & $U^{12}$ & $U^{13}$ & $U^{23}$ \\
\hline Pd1 & $0.0096(8)$ & $0.0087(14)$ & $0.0063(5)$ & $0.0044(7)$ & $0.0006(5)$ & $0.0012(11)$ \\
Pd2 & $0.0096(7)$ & $0.0076(14)$ & $0.0052(4)$ & $0.0038(7)$ & $-0.0002(5)$ & $-0.0003(10)$ \\
Pd3 & $0.0104(7)$ & $0.0104(7)$ & $0.0062(5)$ & $0.0028(13)$ & $0.0005(6)$ & $-0.0005(6)$ \\
Pd4 & $0.0100(8)$ & $0.0122(6)$ & $0.0072(3)$ & $0.0046(8)$ & $0.0002(6)$ & $0.0011(4)$ \\
Pd5 & $0.0143(8)$ & $0.0093(7)$ & $0.0060(3)$ & $0.0074(5)$ & $-0.0004(5)$ & $-0.0002(6)$ \\
Pd6 & $0.0116(8)$ & $0.0116(8)$ & $0.0137(4)$ & $0.0056(5)$ & $0.0001(6)$ & $0.0005(6)$ \\
Pd7 & $0.0087(7)$ & $0.0089(5)$ & $0.0096(3)$ & $0.0049(6)$ & $0.0004(6)$ & $0.0007(4)$ \\
Pd8 & $0.0091(5)$ & $0.0101(7)$ & $0.0099(3)$ & $0.0053(6)$ & $-0.0008(4)$ & $0.0005(6)$ \\
Pd9 & $0.0070(6)$ & $0.0070(6)$ & $0.0060(4)$ & $0.0026(11)$ & $-0.0003(5)$ & $0.0003(5)$ \\
Sn1 & $0.0070(6)$ & $0.0070(6)$ & $0.0060(4)$ & $0.0026(11)$ & $-0.0003(5)$ & $0.0003(5)$ \\
Sn2 & $0.0081(7)$ & $0.0138(4)$ & $0.0076(3)$ & $0.0074(7)$ & $0.0014(5)$ & $0.0019(3)$ \\
Sn3 & $0.0080(7)$ & $0.0069(7)$ & $0.0070(4)$ & $0.0015(3)$ & $0.0001(5)$ & $-0.0004(5)$ \\
Sn4 & $0.0079(4)$ & $0.0072(4)$ & $0.0081(3)$ & $0.0018(4)$ & $0.0002(3)$ & $-0.0001(3)$ \\
Sn5 & $0.0123(4)$ & $0.0089(7)$ & $0.0065(3)$ & $0.0070(7)$ & $-0.0009(2)$ & $-0.0008(5)$ \\
& & & & & &
\end{tabular}

Geometric parameters $(\AA, \stackrel{\circ}{)})$

\begin{tabular}{llll}
\hline $\mathrm{Pd} 1-\mathrm{Sn} 5^{\mathrm{i}}$ & $2.7259(18)$ & $\mathrm{Pd} 5-\mathrm{Pd} 8^{\mathrm{ix}}$ & $2.933(3)$ \\
$\mathrm{Pd} 1-\mathrm{Sn} 5^{\mathrm{ii}}$ & $2.7259(18)$ & $\mathrm{Pd} 5-\mathrm{Sn} 4$ & $2.967(2)$ \\
$\mathrm{Pd} 1-\mathrm{Sn} 2^{\mathrm{iii}}$ & $2.741(4)$ & $\mathrm{Pd} 6-\mathrm{Sn} 4$ & $2.6407(19)$ \\
$\mathrm{Pd} 1-\mathrm{Sn} 2$ & $2.741(4)$ & $\mathrm{Pd} 6-\mathrm{Sn} 2$ & $2.707(2)$
\end{tabular}




\begin{tabular}{|c|c|c|c|}
\hline $\mathrm{Pd} 1-\mathrm{Pd} 3$ & $2.8168(1)$ & Pd6-Sn5 & $2.715(2)$ \\
\hline $\mathrm{Pd} 1-\mathrm{Pd} 3^{\mathrm{iv}}$ & $2.8168(1)$ & $\mathrm{Pd} 6-\mathrm{Pd} 9$ & $2.7553(13)$ \\
\hline $\mathrm{Pd} 1-\mathrm{Sn} 4^{\mathrm{v}}$ & $2.875(3)$ & Pd6-Sn3 ${ }^{\text {viii }}$ & $2.8242(13)$ \\
\hline $\mathrm{Pd} 1-\mathrm{Sn} 4^{\mathrm{vi}}$ & $2.875(3)$ & $\mathrm{Pd} 6-\mathrm{Sn} 3^{\mathrm{ix}}$ & $2.8782(13)$ \\
\hline $\mathrm{Pd} 1-\mathrm{Pd} 8$ & $2.9563(18)$ & $\mathrm{Pd} 6-\mathrm{Pd} 5^{\mathrm{xv}}$ & $2.910(4)$ \\
\hline $\mathrm{Pd} 1-\mathrm{Pd} 8^{\mathrm{iii}}$ & $2.9563(18)$ & $\mathrm{Pd} 6$-Pd4 ${ }^{\text {viii }}$ & 3.017 (4) \\
\hline $\mathrm{Pd} 1-\mathrm{Pd} 7^{\mathrm{ii}}$ & 3.015 (4) & $\mathrm{Pd} 7-\mathrm{Sn} 4^{\mathrm{xvi}}$ & $2.6532(16)$ \\
\hline $\mathrm{Pd} 1-\mathrm{Pd} 7^{\mathrm{i}}$ & $3.015(4)$ & $\mathrm{Pd} 7-\mathrm{Sn} 3$ & $2.746(3)$ \\
\hline $\mathrm{Pd} 2-\mathrm{Sn} 3^{\mathrm{vii}}$ & $2.7264(19)$ & $\mathrm{Pd} 7-\mathrm{Pd} 9$ & $2.7515(19)$ \\
\hline $\mathrm{Pd} 2-\mathrm{Sn} 3^{\text {viii }}$ & $2.7264(19)$ & $\mathrm{Pd} 7-\mathrm{Sn} 5$ & $2.755(2)$ \\
\hline $\mathrm{Pd} 2-\mathrm{Sn} 2^{\mathrm{iii}}$ & $2.738(2)$ & $\mathrm{Pd} 7-\mathrm{Sn} 5^{\text {ix }}$ & $2.8188(11)$ \\
\hline $\mathrm{Pd} 2-\mathrm{Sn} 2$ & $2.738(2)$ & $\mathrm{Pd} 7-\mathrm{Sn} 4^{\mathrm{ii}}$ & $2.8603(9)$ \\
\hline $\mathrm{Pd} 2-\mathrm{Pd} 5$ & $2.8325(7)$ & $\mathrm{Pd} 7-\mathrm{Pd} 5^{\mathrm{xvi}}$ & $2.882(3)$ \\
\hline $\mathrm{Pd} 2-\mathrm{Pd} 5^{\mathrm{iii}}$ & $2.8325(7)$ & $\mathrm{Pd} 7-\mathrm{Pd} 3^{\mathrm{xvii}}$ & $2.884(4)$ \\
\hline $\mathrm{Pd} 2-\mathrm{Sn} 4^{4 i i}$ & 2.8554 (19) & $\mathrm{Pd} 7-\mathrm{Pd} 2^{\mathrm{xvi}}$ & $3.006(2)$ \\
\hline $\mathrm{Pd} 2-\mathrm{Sn} 4$ & $2.8554(19)$ & $\mathrm{Pd} 7-\mathrm{Pd} 1^{\mathrm{xvii}}$ & $3.014(4)$ \\
\hline $\mathrm{Pd} 2-\mathrm{Pd} 6$ & $2.9705(19)$ & $\mathrm{Pd} 8-\mathrm{Sn} 4^{\mathrm{vi}}$ & $2.6552(18)$ \\
\hline $\mathrm{Pd} 2-\mathrm{Pd} 6^{\mathrm{iii}}$ & $2.9705(19)$ & $\mathrm{Pd} 8-\mathrm{Sn} 3$ & $2.708(3)$ \\
\hline $\mathrm{Pd} 2-\mathrm{Pd} 7^{\mathrm{vii}}$ & $3.006(2)$ & $\mathrm{Pd} 8-\mathrm{Sn} 2$ & $2.720(2)$ \\
\hline $\mathrm{Pd} 2-\mathrm{Pd} 7^{\text {viii }}$ & $3.006(2)$ & $\mathrm{Pd} 8-\mathrm{Sn} 5^{\mathrm{ii}}$ & $2.7802(11)$ \\
\hline $\mathrm{Pd} 3-\mathrm{Sn} 2$ & $2.738(3)$ & $\mathrm{Pd} 8-\mathrm{Pd} 9$ & $2.7853(18)$ \\
\hline $\mathrm{Pd} 3-\mathrm{Sn} 2^{\mathrm{ix}}$ & $2.738(3)$ & $\mathrm{Pd} 8-\mathrm{Sn} 2^{\mathrm{ix}}$ & $2.8279(11)$ \\
\hline $\mathrm{Pd} 3-\mathrm{Sn} 5^{\mathrm{x}}$ & $2.811(3)$ & $\mathrm{Pd} 8-\mathrm{Pd} 5^{\mathrm{ix}}$ & $2.933(3)$ \\
\hline $\mathrm{Pd} 3-\mathrm{Sn} 5^{\mathrm{i}}$ & $2.811(3)$ & $\mathrm{Pd} 8-\mathrm{Pd} 4^{\mathrm{ii}}$ & $2.973(2)$ \\
\hline $\mathrm{Pd} 3-\mathrm{Pd} 1^{\mathrm{xi}}$ & $2.8167(1)$ & $\mathrm{Pd} 9-\mathrm{Pd} 7^{\text {ix }}$ & $2.7515(19)$ \\
\hline$P d 3-P d 7^{x}$ & $2.884(4)$ & $\mathrm{Pd} 9-\mathrm{Pd} 6^{\mathrm{ix}}$ & $2.7553(13)$ \\
\hline $\mathrm{Pd} 3-\mathrm{Pd} 7^{\mathrm{i}}$ & $2.884(4)$ & $\mathrm{Pd} 9-\mathrm{Pd} 4^{\mathrm{ix}}$ & $2.7630(7)$ \\
\hline $\mathrm{Pd} 3-\mathrm{Pd} 8^{\mathrm{ix}}$ & $2.932(4)$ & $\mathrm{Pd} 9-\mathrm{Pd} 8^{\mathrm{ix}}$ & $2.7853(18)$ \\
\hline $\mathrm{Pd} 3-\mathrm{Pd} 8$ & $2.932(4)$ & $\mathrm{Pd} 9-\mathrm{Sn} 2$ & $3.2738(16)$ \\
\hline $\mathrm{Pd} 3-\mathrm{Sn} 4^{\mathrm{vi}}$ & $2.9611(11)$ & $\mathrm{Pd} 9-\mathrm{Sn} 2^{\mathrm{ix}}$ & $3.2738(16)$ \\
\hline $\mathrm{Pd} 3-\mathrm{Sn} 4^{\mathrm{xii}}$ & $2.9611(11)$ & $\mathrm{Sn} 2-\mathrm{Pd} 8^{\mathrm{ix}}$ & $2.8279(11)$ \\
\hline $\mathrm{Pd} 4-\mathrm{Sn} 3^{\text {viii }}$ & $2.732(4)$ & $\mathrm{Sn} 2-\mathrm{Sn} 2^{\mathrm{iii}}$ & $3.2924(15)$ \\
\hline $\mathrm{Pd} 4-\mathrm{Sn} 5^{\mathrm{ii}}$ & $2.742(2)$ & $\mathrm{Sn} 3-\mathrm{Pd} 2^{\mathrm{xvi}}$ & $2.7263(19)$ \\
\hline $\mathrm{Pd} 4-\mathrm{Pd} 9$ & $2.7629(7)$ & $\mathrm{Sn} 3-\mathrm{Pd} 4^{\mathrm{ii}}$ & $2.732(4)$ \\
\hline $\mathrm{Pd} 4-\mathrm{Pd} 7$ & $2.805(3)$ & $\mathrm{Sn} 3-\mathrm{Pd} 5^{\mathrm{ix}}$ & $2.781(4)$ \\
\hline $\mathrm{Pd} 4-\mathrm{Pd} 8$ & $2.820(2)$ & $\mathrm{Sn} 3-\mathrm{Pd} 5^{\mathrm{xvi}}$ & $2.797(4)$ \\
\hline $\mathrm{Pd} 4-\mathrm{Pd} 6$ & $2.821(3)$ & $\mathrm{Sn} 3-\mathrm{Pd} 6^{\mathrm{ii}}$ & $2.8242(13)$ \\
\hline $\mathrm{Pd} 4-\mathrm{Sn} 4^{\mathrm{ii}}$ & $2.823(2)$ & $\operatorname{Sn} 3-\mathrm{Pd} 6^{\mathrm{ix}}$ & $2.8783(13)$ \\
\hline $\mathrm{Pd} 4-\mathrm{Pd} 5^{\mathrm{xiii}}$ & $2.8558(9)$ & $\mathrm{Sn} 4-\mathrm{Pd} 7^{\mathrm{vii}}$ & $2.6531(16)$ \\
\hline $\mathrm{Pd} 4-\mathrm{Pd} 8^{\mathrm{viii}}$ & $2.973(2)$ & $\mathrm{Sn} 4-\mathrm{Pd} 8^{\mathrm{xviii}}$ & $2.6552(18)$ \\
\hline $\mathrm{Pd} 4-\mathrm{Pd} 6^{\mathrm{ii}}$ & 3.017 (4) & $\mathrm{Sn} 4-\mathrm{Pd} 4^{\text {viii }}$ & $2.823(2)$ \\
\hline $\mathrm{Pd} 4-\mathrm{Sn} 5$ & $3.1621(19)$ & $\mathrm{Sn} 4-\mathrm{Pd} 7^{\mathrm{viii}}$ & $2.8604(9)$ \\
\hline $\mathrm{Pd} 5-\mathrm{Sn} 2$ & $2.740(3)$ & $\mathrm{Sn} 4-\mathrm{Pd} 1^{\mathrm{xviii}}$ & $2.875(3)$ \\
\hline $\mathrm{Pd} 5-\mathrm{Sn} 5^{\mathrm{xii}}$ & $2.772(3)$ & $\mathrm{Sn} 4-\mathrm{Pd} 5^{\mathrm{xv}}$ & $2.900(2)$ \\
\hline $\mathrm{Pd} 5-\mathrm{Sn} 3^{\mathrm{ix}}$ & $2.781(4)$ & $\mathrm{Sn} 4-\mathrm{Pd} 3^{\mathrm{xviii}}$ & $2.9611(11)$ \\
\hline $\mathrm{Pd} 5-\mathrm{Sn} 3^{\mathrm{vii}}$ & $2.797(4)$ & $\mathrm{Sn} 5-\mathrm{Pd} 1^{\mathrm{xvii}}$ & $2.7258(18)$ \\
\hline $\mathrm{Pd} 5-\mathrm{Pd} 4^{\mathrm{xiv}}$ & $2.8557(9)$ & $\mathrm{Sn} 5-\mathrm{Pd} 4^{\mathrm{viii}}$ & $2.742(2)$ \\
\hline $\mathrm{Pd} 5-\mathrm{Pd} 7^{\mathrm{vii}}$ & $2.882(3)$ & $\mathrm{Sn} 5-\mathrm{Pd} 5^{\mathrm{xv}}$ & $2.772(3)$ \\
\hline
\end{tabular}




\begin{tabular}{|c|c|c|c|}
\hline $\mathrm{Pd} 5-\mathrm{Sn} 4^{\mathrm{xii}}$ & $2.900(2)$ & $\mathrm{Sn} 5-\mathrm{Pd} 8^{\text {viii }}$ & $2.7803(11)$ \\
\hline $\mathrm{Pd} 5-\mathrm{Pd} 6^{\mathrm{xii}}$ & $2.910(4)$ & $\mathrm{Sn} 5-\mathrm{Pd} 3^{\mathrm{xvii}}$ & $2.811(3)$ \\
\hline $\mathrm{Pd} 5-\mathrm{Pd} 6$ & $2.932(4)$ & $\mathrm{Sn} 5-\mathrm{Pd} 7^{\mathrm{ix}}$ & $2.8188(11)$ \\
\hline 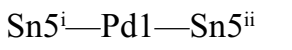 & $164.96(17)$ & $\mathrm{Sn} 2-\mathrm{Pd} 6-\mathrm{Pd} 5^{\mathrm{xv}}$ & $151.78(6)$ \\
\hline $\mathrm{Sn} 5^{\mathrm{i}}-\mathrm{Pd} 1-\mathrm{Sn} 2^{\mathrm{iii}}$ & $83.14(7)$ & $\mathrm{Sn} 5-\mathrm{Pd} 6-\mathrm{Pd} 5^{\mathrm{xv}}$ & $58.92(6)$ \\
\hline $\mathrm{Sn} 5^{\mathrm{ii}}-\mathrm{Pd} 1-\mathrm{Sn} 2^{\mathrm{iii}}$ & $84.84(8)$ & $\mathrm{Pd} 9-\mathrm{Pd} 6-\mathrm{Pd} 5^{\mathrm{xv}}$ & $101.06(7)$ \\
\hline 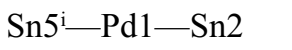 & $84.84(8)$ & $\mathrm{Pd} 4-\mathrm{Pd} 6-\mathrm{Pd} 5^{\mathrm{xv}}$ & $128.45(10)$ \\
\hline $\mathrm{Sn} 5^{\mathrm{ii}}-\mathrm{Pd} 1-\mathrm{Sn} 2$ & $83.14(7)$ & $\mathrm{Sn} 3^{\mathrm{viii}}-\mathrm{Pd} 6-\mathrm{Pd} 5^{\mathrm{xv}}$ & $123.49(7)$ \\
\hline $\mathrm{Sn} 2 \mathrm{iii}-\mathrm{Pd} 1-\mathrm{Sn} 2$ & $73.82(12)$ & $\mathrm{Sn} 3^{\mathrm{ix}}-\mathrm{Pd} 6-\mathrm{Pd} 5^{\mathrm{xv}}$ & $57.78(6)$ \\
\hline 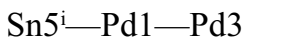 & $60.92(7)$ & $\mathrm{Sn} 4-\mathrm{Pd} 6-\mathrm{Pd} 5$ & $64.09(5)$ \\
\hline $\mathrm{Sn} 5^{\mathrm{ii}}-\mathrm{Pd} 1-\mathrm{Pd} 3$ & $119.10(9)$ & $\mathrm{Sn} 2-\mathrm{Pd} 6-\mathrm{Pd} 5$ & $57.98(6)$ \\
\hline $\mathrm{Sn} 2^{\mathrm{iii}}-\mathrm{Pd} 1-\mathrm{Pd} 3$ & $121.12(13)$ & $\mathrm{Sn} 5-\mathrm{Pd} 6-\mathrm{Pd} 5$ & $153.21(6)$ \\
\hline $\mathrm{Sn} 2-\mathrm{Pd} 1-\mathrm{Pd} 3$ & $59.00(9)$ & $\mathrm{Pd} 9-\mathrm{Pd} 6-\mathrm{Pd} 5$ & $101.37(7)$ \\
\hline $\mathrm{Sn} 5^{\mathrm{i}}-\mathrm{Pd} 1-\mathrm{Pd} 3^{\mathrm{iv}}$ & $119.10(9)$ & $\mathrm{Pd} 4-\mathrm{Pd} 6-\mathrm{Pd} 5$ & $131.43(10)$ \\
\hline $\mathrm{Sn} 5^{\mathrm{ii}}-\mathrm{Pd} 1-\mathrm{Pd} 3^{\mathrm{iv}}$ & $60.91(7)$ & 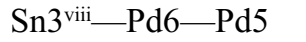 & $113.13(8)$ \\
\hline $\mathrm{Sn} 2^{\mathrm{iii}}-\mathrm{Pd} 1-\mathrm{Pd} 3^{\mathrm{iv}}$ & $59.00(9)$ & $\mathrm{Sn} 3^{\mathrm{ix}}-\mathrm{Pd} 6-\mathrm{Pd} 5$ & $57.19(6)$ \\
\hline $\mathrm{Sn} 2-\mathrm{Pd} 1-\mathrm{Pd} 3^{\mathrm{iv}}$ & $121.11(13)$ & $\mathrm{Pd} 5^{\mathrm{xv}}-\mathrm{Pd} 6-\mathrm{Pd} 5$ & $97.37(4)$ \\
\hline $\mathrm{Pd} 3-\mathrm{Pd} 1-\mathrm{Pd} 3^{\mathrm{iv}}$ & $179.9(2)$ & $\mathrm{Sn} 4-\mathrm{Pd} 6-\mathrm{Pd} 2$ & $60.84(6)$ \\
\hline 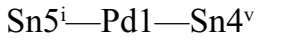 & $86.43(6)$ & $\mathrm{Sn} 2-\mathrm{Pd} 6-\mathrm{Pd} 2$ & $57.45(7)$ \\
\hline $\mathrm{Sn} 5^{\mathrm{ii}}-\mathrm{Pd} 1-\mathrm{Sn} 4^{\mathrm{v}}$ & $105.28(8)$ & $\mathrm{Sn} 5-\mathrm{Pd} 6-\mathrm{Pd} 2$ & $144.58(5)$ \\
\hline 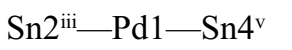 & $103.97(3)$ & $\mathrm{Pd} 9-\mathrm{Pd} 6-\mathrm{Pd} 2$ & $130.92(9)$ \\
\hline $\mathrm{Sn} 2-\mathrm{Pd} 1-\mathrm{Sn} 4^{\mathrm{v}}$ & $171.20(4)$ & $\mathrm{Pd} 4-\mathrm{Pd} 6-\mathrm{Pd} 2$ & $99.73(7)$ \\
\hline $\mathrm{Pd} 3-\mathrm{Pd} 1-\mathrm{Sn} 4^{\mathrm{v}}$ & $117.20(15)$ & 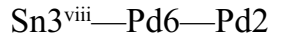 & $56.06(5)$ \\
\hline $\mathrm{Pd} 3^{\mathrm{iv}}-\mathrm{Pd} 1-\mathrm{Sn} 4^{\mathrm{v}}$ & $62.69(4)$ & $\mathrm{Sn} 3^{\mathrm{ix}}-\mathrm{Pd} 6-\mathrm{Pd} 2$ & $113.74(8)$ \\
\hline $\mathrm{Sn} 5^{\mathrm{i}}-\mathrm{Pd} 1-\mathrm{Sn} 4^{\mathrm{vi}}$ & $105.27(8)$ & $\mathrm{Pd} 5^{\mathrm{xv}}-\mathrm{Pd} 6-\mathrm{Pd} 2$ & $123.60(8)$ \\
\hline $\mathrm{Sn} 5^{\mathrm{ii}}-\mathrm{Pd} 1-\mathrm{Sn} 4^{\mathrm{vi}}$ & $86.43(6)$ & $\mathrm{Pd} 5-\mathrm{Pd} 6-\mathrm{Pd} 2$ & $57.35(5)$ \\
\hline $\mathrm{Sn} 2^{\mathrm{iii}}-\mathrm{Pd} 1-\mathrm{Sn} 4^{\mathrm{vi}}$ & $171.20(4)$ & $\mathrm{Sn} 4-\mathrm{Pd} 6-\mathrm{Pd} 4{ }^{\mathrm{viii}}$ & $59.42(5)$ \\
\hline $\mathrm{Sn} 2-\mathrm{Pd} 1-\mathrm{Sn} 4^{\mathrm{vi}}$ & $103.97(3)$ & $\mathrm{Sn} 2-\mathrm{Pd} 6-\mathrm{Pd} 4^{\mathrm{viii}}$ & $145.74(7)$ \\
\hline $\mathrm{Pd} 3-\mathrm{Pd} 1-\mathrm{Sn} 4^{\mathrm{vi}}$ & $62.69(4)$ & $\mathrm{Sn} 5-\mathrm{Pd} 6-\mathrm{Pd} 4^{\mathrm{viii}}$ & $56.86(6)$ \\
\hline $\mathrm{Pd} 3^{\mathrm{iv}}-\mathrm{Pd} 1-\mathrm{Sn} 4^{\mathrm{vi}}$ & $117.20(15)$ & $\mathrm{Pd} 9-\mathrm{Pd} 6-\mathrm{Pd} 4{ }^{\mathrm{viii}}$ & $130.73(10)$ \\
\hline $\mathrm{Sn} 4^{\mathrm{v}}-\mathrm{Pd} 1-\mathrm{Sn} 4^{\mathrm{vi}}$ & $79.49(11)$ & $\mathrm{Pd} 4-\mathrm{Pd} 6-\mathrm{Pd} 4^{\text {viii }}$ & $97.46(5)$ \\
\hline $\mathrm{Sn} 55^{\mathrm{i}-\mathrm{Pd} 1-\mathrm{Pd} 8}$ & $120.98(4)$ & 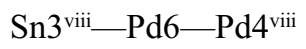 & $65.93(5)$ \\
\hline $\mathrm{Sn} 5^{\mathrm{ii}-\mathrm{Pd} 1-\mathrm{Pd} 8}$ & $58.42(3)$ & $\mathrm{Sn} 3^{\mathrm{ix}}-\mathrm{Pd} 6-\mathrm{Pd} 4^{\mathrm{viii}}$ & $114.46(8)$ \\
\hline $\mathrm{Sn} 2{ }^{\mathrm{iii}}-\mathrm{Pd} 1-\mathrm{Pd} 8$ & $119.43(12)$ & $\mathrm{Pd} 5^{\mathrm{xv}}-\mathrm{Pd} 6-\mathrm{Pd} 4^{\mathrm{viii}}$ & $57.57(6)$ \\
\hline $\mathrm{Sn} 2-\mathrm{Pd} 1-\mathrm{Pd} 8$ & $56.89(5)$ & $\mathrm{Pd} 5-\mathrm{Pd} 6-\mathrm{Pd} 4^{\mathrm{viii}}$ & $123.51(7)$ \\
\hline $\mathrm{Pd} 3-\mathrm{Pd} 1-\mathrm{Pd} 8$ & $60.99(8)$ & $\mathrm{Pd} 2-\mathrm{Pd} 6-\mathrm{Pd} 4{ }^{\mathrm{viii}}$ & $93.11(6)$ \\
\hline $\mathrm{Pd} 3^{\mathrm{iv}}-\mathrm{Pd} 1-\mathrm{Pd} 8$ & $119.01(8)$ & $\mathrm{Sn} 4^{\mathrm{xvi}}-\mathrm{Pd} 7-\mathrm{Sn} 3$ & $110.53(8)$ \\
\hline $\mathrm{Sn} 4^{\mathrm{v}}-\mathrm{Pd} 1-\mathrm{Pd} 8$ & $129.67(13)$ & $\mathrm{Sn} 4^{\mathrm{xvi}}-\mathrm{Pd} 7-\mathrm{Pd} 9$ & $157.08(4)$ \\
\hline 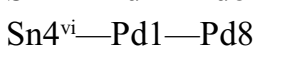 & $54.16(4)$ & $\mathrm{Sn} 3-\mathrm{Pd} 7-\mathrm{Pd} 9$ & $73.74(5)$ \\
\hline $\mathrm{Sn} 5^{\mathrm{i}}-\mathrm{Pd} 1-\mathrm{Pd} 8^{\mathrm{iii}}$ & $58.42(3)$ & $\mathrm{Sn} 4^{\mathrm{xvi}}-\mathrm{Pd} 7-\mathrm{Sn} 5$ & $110.81(8)$ \\
\hline $\mathrm{Sn} 5^{\mathrm{ii}}-\mathrm{Pd} 1-\mathrm{Pd} 8^{\mathrm{iii}}$ & $120.98(4)$ & $\mathrm{Sn} 3-\mathrm{Pd} 7-\mathrm{Sn} 5$ & $136.65(6)$ \\
\hline $\mathrm{Sn} 2^{\mathrm{iii}}-\mathrm{Pd} 1-\mathrm{Pd} 8^{\mathrm{iii}}$ & $56.89(5)$ & $\mathrm{Pd} 9-\mathrm{Pd} 7-\mathrm{Sn} 5$ & $73.41(4)$ \\
\hline $\mathrm{Sn} 2-\mathrm{Pd} 1-\mathrm{Pd} 8^{\mathrm{iii}}$ & $119.43(12)$ & $\mathrm{Sn} 4^{\mathrm{xvi}}-\mathrm{Pd} 7-\mathrm{Pd} 4$ & $143.30(5)$ \\
\hline $\mathrm{Pd} 3-\mathrm{Pd} 1-\mathrm{Pd} 8^{\mathrm{iii}}$ & $119.01(8)$ & $\mathrm{Sn} 3-\mathrm{Pd} 7-\mathrm{Pd} 4$ & $69.96(7)$ \\
\hline $\mathrm{Pd} 3^{\mathrm{iv}}-\mathrm{Pd} 1-\mathrm{Pd} 8^{\mathrm{iii}}$ & $60.99(8)$ & $\mathrm{Pd} 9-\mathrm{Pd} 7-\mathrm{Pd} 4$ & $59.62(4)$ \\
\hline $\mathrm{Sn} 4^{\mathrm{v}}-\mathrm{Pd} 1-\mathrm{Pd} 8^{\mathrm{iii}}$ & $54.15(4)$ & $\mathrm{Sn} 5-\mathrm{Pd} 7-\mathrm{Pd} 4$ & $69.31(7)$ \\
\hline $\mathrm{Sn} 4^{\mathrm{vi}}-\mathrm{Pd} 1-\mathrm{Pd} 8^{\mathrm{iii}}$ & $129.67(13)$ & $\mathrm{Sn} 4^{\mathrm{xvi}}-\mathrm{Pd} 7-\mathrm{Sn} 5^{\mathrm{ix}}$ & $84.66(4)$ \\
\hline
\end{tabular}




\begin{tabular}{|c|c|}
\hline $\mathrm{Pd} 8-\mathrm{Pd} 1-\mathrm{Pd} 8^{\mathrm{iii}}$ & $176.07(17)$ \\
\hline 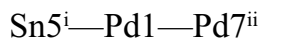 & $137.27(13)$ \\
\hline $\mathrm{Sn} 5^{\mathrm{ii}}-\mathrm{Pd} 1-\mathrm{Pd} 7^{\mathrm{ii}}$ & $57.09(5)$ \\
\hline 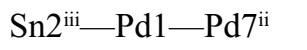 & $117.33(4)$ \\
\hline $\mathrm{Sn} 2-\mathrm{Pd} 1-\mathrm{Pd} 7^{\mathrm{ii}}$ & $135.21(3)$ \\
\hline $\mathrm{Pd} 3-\mathrm{Pd} 1-\mathrm{Pd} 7^{\mathrm{ii}}$ & $120.73(11)$ \\
\hline $\mathrm{Pd} 3^{\mathrm{iv}}-\mathrm{Pd} 1-\mathrm{Pd} 7^{\mathrm{ii}}$ & $59.16(10)$ \\
\hline $\mathrm{Sn} 4^{\mathrm{v}}-\mathrm{Pd} 1-\mathrm{Pd} 7^{\mathrm{ii}}$ & $53.49(7)$ \\
\hline $\mathrm{Sn} 4^{\mathrm{vi}}-\mathrm{Pd} 1-\mathrm{Pd} 7^{\mathrm{ii}}$ & $58.06(7)$ \\
\hline $\mathrm{Pd} 8-\mathrm{Pd} 1-\mathrm{Pd} 7^{\mathrm{ii}}$ & $83.04(6)$ \\
\hline 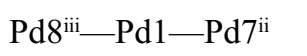 & $99.87(7)$ \\
\hline $\mathrm{Sn} 5^{\mathrm{i}}-\mathrm{Pd} 1-\mathrm{Pd} 7^{\mathrm{i}}$ & $57.09(5)$ \\
\hline $\mathrm{Sn} 5^{{ }^{\mathrm{ii}}-\mathrm{Pd} 1} 1-\mathrm{Pd} 7^{\mathrm{i}}$ & $137.27(13)$ \\
\hline 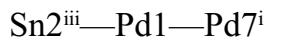 & $135.21(3)$ \\
\hline $\mathrm{Sn} 2-\mathrm{Pd} 1-\mathrm{Pd} 7^{\mathrm{i}}$ & $117.33(4)$ \\
\hline $\mathrm{Pd} 3-\mathrm{Pd} 1-\mathrm{Pd} 7^{\mathrm{i}}$ & $59.16(10)$ \\
\hline$P d 3^{i v}-P d 1-P d 7^{i}$ & $120.73(11)$ \\
\hline $\mathrm{Sn} 4^{\mathrm{v}}-\mathrm{Pd} 1-\mathrm{Pd} 7^{\mathrm{i}}$ & $58.06(7)$ \\
\hline 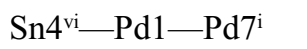 & $53.49(7)$ \\
\hline $\mathrm{Pd} 8-\mathrm{Pd} 1-\mathrm{Pd} 7^{\mathrm{i}}$ & $99.87(7)$ \\
\hline $\mathrm{Pd} 8^{\mathrm{iii}}-\mathrm{Pd} 1-\mathrm{Pd} 7^{\mathrm{i}}$ & $83.04(6)$ \\
\hline 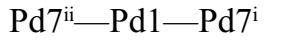 & $86.07(12)$ \\
\hline $\mathrm{Sn} 3^{\mathrm{vii}}-\mathrm{Pd} 2-\mathrm{Sn} 3^{\mathrm{viii}}$ & $164.85(10)$ \\
\hline 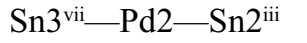 & $83.18(5)$ \\
\hline 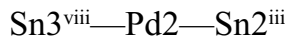 & $84.72(4)$ \\
\hline 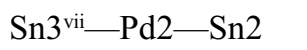 & $84.73(4)$ \\
\hline 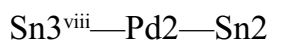 & $83.18(5)$ \\
\hline 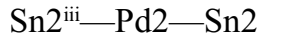 & $73.91(8)$ \\
\hline $\mathrm{Sn} 3^{\mathrm{vii}}-\mathrm{Pd} 2-\mathrm{Pd} 5$ & $60.38(8)$ \\
\hline 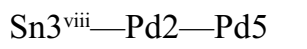 & $119.57(8)$ \\
\hline $\mathrm{Sn} 2^{\mathrm{iii}-\mathrm{Pd} 2-\mathrm{Pd} 5}$ & $120.80(8)$ \\
\hline $\mathrm{Sn} 2-\mathrm{Pd} 2-\mathrm{Pd} 5$ & $58.89(6)$ \\
\hline $\mathrm{Sn} 3^{\mathrm{vii}}-\mathrm{Pd} 2-\mathrm{Pd} 5^{\mathrm{iii}}$ & $119.57(8)$ \\
\hline $\mathrm{Sn} 3^{\mathrm{viii}}-\mathrm{Pd} 2-\mathrm{Pd} 5^{\mathrm{iii}}$ & $60.38(8)$ \\
\hline 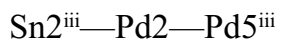 & $58.89(6)$ \\
\hline $\mathrm{Sn} 2-\mathrm{Pd} 2-\mathrm{Pd} 5^{\mathrm{iii}}$ & $120.80(8)$ \\
\hline $\mathrm{Pd} 5-\mathrm{Pd} 2-\mathrm{Pd} 5^{\mathrm{iii}}$ & $179.68(12)$ \\
\hline $\mathrm{Sn} 3^{\mathrm{vii}}-\mathrm{Pd} 2-\mathrm{Sn} 4^{\mathrm{iii}}$ & $86.47(4)$ \\
\hline $\mathrm{Sn} 3^{\mathrm{viii}}-\mathrm{Pd} 2-\mathrm{Sn} 4^{\mathrm{iii}}$ & $105.28(5)$ \\
\hline $\mathrm{Sn} 2^{\mathrm{iii}}-\mathrm{Pd} 2-\mathrm{Sn} 4^{\mathrm{iii}}$ & $103.61(3)$ \\
\hline $\mathrm{Sn} 2-\mathrm{Pd} 2-\mathrm{Sn} 4^{\mathrm{iii}}$ & $171.07(3)$ \\
\hline $\mathrm{Pd} 5-\mathrm{Pd} 2-\mathrm{Sn} 4^{\mathrm{iii}}$ & $117.39(9)$ \\
\hline $\mathrm{Pd} 5^{\mathrm{iii}}-\mathrm{Pd} 2-\mathrm{Sn} 4^{\mathrm{iii}}$ & $62.89(5)$ \\
\hline $\mathrm{Sn} 3^{\mathrm{vii}}-\mathrm{Pd} 2-\mathrm{Sn} 4$ & $105.27(5)$ \\
\hline 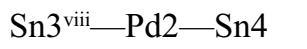 & $86.47(4)$ \\
\hline 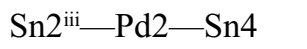 & $171.07(3)$ \\
\hline $\mathrm{Sn} 2-\mathrm{Pd} 2-\mathrm{Sn} 4$ & $103.61(3)$ \\
\hline $\mathrm{Pd} 5-\mathrm{Pd} 2-\mathrm{Sn} 4$ & 62.89 \\
\hline
\end{tabular}

\begin{tabular}{|c|c|}
\hline $\mathrm{Sn} 3-\mathrm{Pd} 7-\mathrm{Sn} 5^{\mathrm{ix}}$ & $97.76(7)$ \\
\hline $\mathrm{Pd} 9-\mathrm{Pd} 7-\mathrm{Sn} 5^{\mathrm{ix}}$ & $72.42(3)$ \\
\hline $\mathrm{Sn} 5-\mathrm{Pd} 7-\mathrm{Sn} 5^{\mathrm{ix}}$ & $98.45(7)$ \\
\hline $\mathrm{Pd} 4-\mathrm{Pd} 7-\mathrm{Sn} 5^{\mathrm{ix}}$ & $132.04(6)$ \\
\hline $\mathrm{Sn} 4^{\mathrm{xvi}}-\mathrm{Pd} 7-\mathrm{Sn} 4^{\mathrm{ii}}$ & $83.53(4)$ \\
\hline $\mathrm{Sn} 3-\mathrm{Pd} 7-\mathrm{Sn} 4^{\mathrm{ii}}$ & $86.01(5)$ \\
\hline $\mathrm{Pd} 9-\mathrm{Pd} 7-\mathrm{Sn} 4^{\mathrm{ii}}$ & $119.39(5)$ \\
\hline $\mathrm{Sn} 5-\mathrm{Pd} 7-\mathrm{Sn} 4^{\mathrm{ii}}$ & $86.17(5)$ \\
\hline $\mathrm{Pd} 4-\mathrm{Pd} 7-\mathrm{Sn} 4^{\mathrm{ii}}$ & $59.77(4)$ \\
\hline $\mathrm{Sn} 5^{\mathrm{ix}}-\mathrm{Pd} 7-\mathrm{Sn} 4^{\mathrm{ii}}$ & $168.19(6)$ \\
\hline $\mathrm{Sn} 4^{\mathrm{xvi}}-\mathrm{Pd} 7-\mathrm{Pd} 5^{\mathrm{xvi}}$ & $64.68(7)$ \\
\hline $\mathrm{Sn} 3-\mathrm{Pd} 7-\mathrm{Pd} 5^{\mathrm{xvi}}$ & $59.54(7)$ \\
\hline $\mathrm{Pd} 9-\mathrm{Pd} 7-\mathrm{Pd} 5^{\mathrm{xvi}}$ & $101.87(7)$ \\
\hline Sn5-Pd7-Pd5 ${ }^{\mathrm{xvi}}$ & $155.91(5)$ \\
\hline $\mathrm{Pd} 4-\mathrm{Pd} 7-\mathrm{Pd} 5^{\mathrm{xvi}}$ & $129.46(10)$ \\
\hline $\mathrm{Sn} 5^{\mathrm{ix}}-\mathrm{Pd} 7-\mathrm{Pd} 5^{\mathrm{xvi}}$ & $58.18(5)$ \\
\hline $\mathrm{Sn} 4{ }^{\mathrm{ii}}-\mathrm{Pd} 7-\mathrm{Pd} 5^{\mathrm{xvi}}$ & $115.64(7)$ \\
\hline $\mathrm{Sn} 4^{\mathrm{xvi}}-\mathrm{Pd} 7-\mathrm{Pd} 3^{\mathrm{xvii}}$ & $64.50(7)$ \\
\hline $\mathrm{Sn} 3-\mathrm{Pd} 7-\mathrm{Pd} 3^{\mathrm{xvii}}$ & $156.01(5)$ \\
\hline $\mathrm{Pd} 9-\mathrm{Pd} 7-\mathrm{Pd} 3^{\mathrm{xvii}}$ & $102.03(6)$ \\
\hline $\mathrm{Sn} 5-\mathrm{Pd} 7-\mathrm{Pd} 3^{\mathrm{xvii}}$ & $59.74(6)$ \\
\hline $\mathrm{Pd} 4-\mathrm{Pd} 7-\mathrm{Pd} 3^{\mathrm{xvii}}$ & $129.03(9)$ \\
\hline $\mathrm{Sn} 5^{\mathrm{ix}}-\mathrm{Pd} 7-\mathrm{Pd} 3^{\mathrm{xvii}}$ & $59.05(6)$ \\
\hline $\mathrm{Sn} 4^{\mathrm{ii}}-\mathrm{Pd} 7-\mathrm{Pd} 3^{\mathrm{xvii}}$ & $115.50(7)$ \\
\hline $\mathrm{Pd} 5^{\mathrm{xvi}}-\mathrm{Pd} 7-\mathrm{Pd} 3^{\mathrm{xvii}}$ & $99.51(4)$ \\
\hline $\mathrm{Sn} 4^{\mathrm{xvi}}-\mathrm{Pd} 7-\mathrm{Pd} 2^{\mathrm{xvi}}$ & $60.21(4)$ \\
\hline $\mathrm{Sn} 3-\mathrm{Pd} 7-\mathrm{Pd} 2^{\mathrm{xvi}}$ & $56.36(6)$ \\
\hline $\mathrm{Pd} 9-\mathrm{Pd} 7-\mathrm{Pd} 2^{\mathrm{xvi}}$ & $129.97(8)$ \\
\hline $\mathrm{Sn} 5-\mathrm{Pd} 7-\mathrm{Pd} 2^{\mathrm{xvi}}$ & $143.17(5)$ \\
\hline $\mathrm{Pd} 4-\mathrm{Pd} 7-\mathrm{Pd} 2^{\mathrm{xvi}}$ & $96.79(7)$ \\
\hline $\mathrm{Sn} 5^{\mathrm{ix}}-\mathrm{Pd} 7-\mathrm{Pd} 2^{\mathrm{xvi}}$ & $114.87(7)$ \\
\hline $\mathrm{Sn} 4^{\mathrm{ii}}-\mathrm{Pd} 7-\mathrm{Pd} 2^{\mathrm{xvi}}$ & $58.19(4)$ \\
\hline $\mathrm{Pd} 5^{\mathrm{xvi}}-\mathrm{Pd} 7-\mathrm{Pd} 2^{\mathrm{xvi}}$ & $57.46(4)$ \\
\hline $\mathrm{Pd} 3^{\mathrm{xvi}}-\mathrm{Pd} 7-\mathrm{Pd} 2^{\mathrm{xvi}}$ & $124.71(7)$ \\
\hline $\mathrm{Sn} 4^{\mathrm{xvi}}-\mathrm{Pd} 7-\mathrm{Pd} 1^{\mathrm{xvii}}$ & $60.56(5)$ \\
\hline $\mathrm{Sn} 3-\mathrm{Pd} 7-\mathrm{Pd} 1^{\mathrm{xvii}}$ & $143.39(6)$ \\
\hline $\mathrm{Pd} 9-\mathrm{Pd} 7-\mathrm{Pd} 1^{\mathrm{xvii}}$ & $129.46(8)$ \\
\hline $\mathrm{Sn} 5-\mathrm{Pd} 7-\mathrm{Pd} 1^{\mathrm{xvii}}$ & $56.17(7)$ \\
\hline $\mathrm{Pd} 4-\mathrm{Pd} 7-\mathrm{Pd} 1^{\mathrm{xvii}}$ & $96.32(5)$ \\
\hline $\mathrm{Sn} 5^{\mathrm{ix}}-\mathrm{Pd} 7-\mathrm{Pd} 1^{\mathrm{xvii}}$ & $115.31(8)$ \\
\hline $\mathrm{Sn} 4^{\mathrm{ii}}-\mathrm{Pd} 7-\mathrm{Pd} 1^{\mathrm{xvii}}$ & $58.52(5)$ \\
\hline $\mathrm{Pd} 5^{\mathrm{xvi}}-\mathrm{Pd} 7-\mathrm{Pd} 1^{\mathrm{xvii}}$ & $125.23(7)$ \\
\hline $\mathrm{Pd} 3^{\mathrm{xvii}}-\mathrm{Pd} 7-\mathrm{Pd} 1^{\mathrm{xvii}}$ & $57.00(6)$ \\
\hline $\mathrm{Pd} 2^{\mathrm{xvi}}-\mathrm{Pd} 7-\mathrm{Pd} 1^{\mathrm{xvii}}$ & $93.78(5)$ \\
\hline $\mathrm{Sn} 4^{\mathrm{vi}}-\mathrm{Pd} 8-\mathrm{Sn} 3$ & $109.23(8)$ \\
\hline $\mathrm{Sn} 4^{\mathrm{vi}}-\mathrm{Pd} 8-\mathrm{Sn} 2$ & $110.81(8)$ \\
\hline $\mathrm{Sn} 3-\mathrm{Pd} 8-\mathrm{Sn} 2$ & $139.66(6)$ \\
\hline $\mathrm{Sn} 4^{\mathrm{vi}}-\mathrm{Pd} 8-\mathrm{Sn} 5^{\mathrm{ii}}$ & $89.76(5)$ \\
\hline
\end{tabular}




\begin{tabular}{|c|c|c|c|}
\hline $\mathrm{Pd} 5^{\mathrm{iii}-\mathrm{Pd} 2-\mathrm{Sn} 4}$ & $117.39(9)$ & $\mathrm{Sn} 3-\mathrm{Pd} 8-\mathrm{Sn} 5^{\mathrm{ii}}$ & $92.94(6)$ \\
\hline 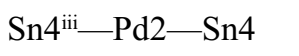 & $80.13(7)$ & $\mathrm{Sn} 2-\mathrm{Pd} 8-\mathrm{Sn} 5^{\mathrm{ii}}$ & $82.52(5)$ \\
\hline $\mathrm{Sn} 3^{\mathrm{vii}-\mathrm{Pd} 2-\mathrm{Pd} 6}$ & $120.09(4)$ & $\mathrm{Sn} 4^{\mathrm{vi}}-\mathrm{Pd} 8-\mathrm{Pd} 9$ & $152.99(4)$ \\
\hline 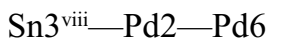 & $59.25(4)$ & $\mathrm{Sn} 3-\mathrm{Pd} 8-\mathrm{Pd} 9$ & $73.79(5)$ \\
\hline $\mathrm{Sn} 2^{\mathrm{iii}-\mathrm{Pd} 2-\mathrm{Pd} 6}$ & $119.55(7)$ & $\mathrm{Sn} 2-\mathrm{Pd} 8-\mathrm{Pd} 9$ & $72.96(4)$ \\
\hline $\mathrm{Sn} 2-\mathrm{Pd} 2-\mathrm{Pd} 6$ & $56.43(4)$ & $\mathrm{Sn} 5^{\mathrm{ii}}-\mathrm{Pd} 8-\mathrm{Pd} 9$ & $117.14(6)$ \\
\hline $\mathrm{Pd} 5-\mathrm{Pd} 2-\mathrm{Pd} 6$ & $60.64(8)$ & $\mathrm{Sn} 4^{\mathrm{vi}}-\mathrm{Pd} 8-\mathrm{Pd} 4$ & $147.89(4)$ \\
\hline $\mathrm{Pd} 5{ }^{\mathrm{iii}}-\mathrm{Pd} 2-\mathrm{Pd} 6$ & $119.35(8)$ & $\mathrm{Sn} 3-\mathrm{Pd} 8-\mathrm{Pd} 4$ & $70.28(8)$ \\
\hline $\mathrm{Sn} 4^{\mathrm{iii}}-\mathrm{Pd} 2-\mathrm{Pd} 6$ & $130.31(7)$ & $\mathrm{Sn} 2-\mathrm{Pd} 8-\mathrm{Pd} 4$ & $73.34(8)$ \\
\hline $\mathrm{Sn} 4-\mathrm{Pd} 2-\mathrm{Pd} 6$ & $53.86(4)$ & $\mathrm{Sn} 5^{\mathrm{ii}}-\mathrm{Pd} 8-\mathrm{Pd} 4$ & $58.64(5)$ \\
\hline $\mathrm{Sn} 3^{\mathrm{vii}}-\mathrm{Pd} 2-\mathrm{Pd} 6^{\mathrm{iii}}$ & $59.25(4)$ & $\mathrm{Pd} 9-\mathrm{Pd} 8-\mathrm{Pd} 4$ & $59.07(4)$ \\
\hline $\mathrm{Sn} 3^{\mathrm{viii}}-\mathrm{Pd} 2-\mathrm{Pd} 6^{\mathrm{iii}}$ & $120.09(4)$ & $\mathrm{Sn} 4^{\mathrm{vi}}-\mathrm{Pd} 8-\mathrm{Sn} 2^{\mathrm{ix}}$ & $81.65(4)$ \\
\hline 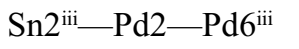 & $56.43(4)$ & $\mathrm{Sn} 3-\mathrm{Pd} 8-\mathrm{Sn} 2^{\mathrm{ix}}$ & $96.03(8)$ \\
\hline $\mathrm{Sn} 2-\mathrm{Pd} 2-\mathrm{Pd} 6^{\mathrm{iii}}$ & $119.56(7)$ & $\mathrm{Sn} 2-\mathrm{Pd} 8-\mathrm{Sn} 2^{\mathrm{ix}}$ & $94.39(7)$ \\
\hline $\mathrm{Pd} 5-\mathrm{Pd} 2-\mathrm{Pd} 6^{\mathrm{iii}}$ & $119.34(8)$ & $\mathrm{Sn} 5^{\mathrm{ii}}-\mathrm{Pd} 8-\mathrm{Sn} 2^{\mathrm{ix}}$ & $169.22(8)$ \\
\hline $\mathrm{Pd} 5^{\mathrm{iii}}-\mathrm{Pd} 2-\mathrm{Pd} 6^{\mathrm{iii}}$ & $60.64(8)$ & $\mathrm{Pd} 9-\mathrm{Pd} 8-\mathrm{Sn} 2^{\mathrm{ix}}$ & $71.35(3)$ \\
\hline $\mathrm{Sn} 4^{\mathrm{iii}}-\mathrm{Pd} 2-\mathrm{Pd} 6^{\mathrm{iii}}$ & $53.86(4)$ & $\mathrm{Pd} 4-\mathrm{Pd} 8-\mathrm{Sn} 2^{\mathrm{ix}}$ & $130.40(5)$ \\
\hline $\mathrm{Sn} 4-\mathrm{Pd} 2-\mathrm{Pd} 6^{\mathrm{iii}}$ & $130.31(7)$ & $\mathrm{Sn} 4{ }^{\mathrm{vi}}-\mathrm{Pd} 8-\mathrm{Pd} 3$ & $63.78(6)$ \\
\hline $\mathrm{Pd} 6-\mathrm{Pd} 2-\mathrm{Pd} 6^{\mathrm{iii}}$ & $175.71(10)$ & $\mathrm{Sn} 3-\mathrm{Pd} 8-\mathrm{Pd} 3$ & $151.91(5)$ \\
\hline $\mathrm{Sn} 3^{\mathrm{vii}}-\mathrm{Pd} 2-\mathrm{Pd} 7^{\mathrm{vii}}$ & $56.98(4)$ & $\mathrm{Sn} 2-\mathrm{Pd} 8-\mathrm{Pd} 3$ & $57.79(6)$ \\
\hline 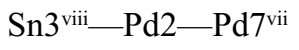 & $137.49(8)$ & $\mathrm{Sn} 5{ }^{\mathrm{ii}}-\mathrm{Pd} 8-\mathrm{Pd} 3$ & $113.52(8)$ \\
\hline 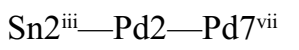 & $135.11(4)$ & $\mathrm{Pd} 9-\mathrm{Pd} 8-\mathrm{Pd} 3$ & $100.42(6)$ \\
\hline $\mathrm{Sn} 2-\mathrm{Pd} 2-\mathrm{Pd} 7^{\mathrm{vii}}$ & $117.19(3)$ & $\mathrm{Pd} 4-\mathrm{Pd} 8-\mathrm{Pd} 3$ & $131.02(11)$ \\
\hline $\mathrm{Pd} 5-\mathrm{Pd} 2-\mathrm{Pd} 7^{\mathrm{vii}}$ & $59.06(6)$ & $\mathrm{Sn} 2^{\mathrm{ix}}-\mathrm{Pd} 8-\mathrm{Pd} 3$ & $56.72(6)$ \\
\hline $\mathrm{Pd} 5^{\mathrm{iii}}-\mathrm{Pd} 2-\mathrm{Pd} 7^{\mathrm{vii}}$ & $121.22(7)$ & 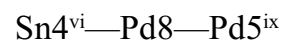 & $62.28(7)$ \\
\hline $\mathrm{Sn} 4^{\mathrm{iii}}-\mathrm{Pd} 2-\mathrm{Pd} 7^{\mathrm{vii}}$ & $58.34(4)$ & $\mathrm{Sn} 3-\mathrm{Pd} 8-\mathrm{Pd} 5^{\mathrm{ix}}$ & $58.92(7)$ \\
\hline $\mathrm{Sn} 4-\mathrm{Pd} 2-\mathrm{Pd} 7^{\mathrm{vii}}$ & $53.75(5)$ & $\mathrm{Sn} 2-\mathrm{Pd} 8-\mathrm{Pd} 5^{\mathrm{ix}}$ & $150.35(5)$ \\
\hline $\mathrm{Pd} 6-\mathrm{Pd} 2-\mathrm{Pd} 7^{\mathrm{vii}}$ & $99.52(4)$ & $\mathrm{Sn} 5^{\mathrm{ii}}-\mathrm{Pd} 8-\mathrm{Pd} 5^{\mathrm{ix}}$ & $124.40(6)$ \\
\hline $\mathrm{Pd} 6{ }^{\mathrm{iii}}-\mathrm{Pd} 2-\mathrm{Pd} 7^{\mathrm{vii}}$ & $83.64(5)$ & $\mathrm{Pd} 9-\mathrm{Pd} 8-\mathrm{Pd} 5^{\mathrm{ix}}$ & $100.63(7)$ \\
\hline $\mathrm{Sn} 3^{\mathrm{vii}}-\mathrm{Pd} 2-\mathrm{Pd} 7^{\mathrm{viii}}$ & $137.49(8)$ & $\mathrm{Pd} 4-\mathrm{Pd} 8-\mathrm{Pd} 5^{\mathrm{ix}}$ & $129.05(12)$ \\
\hline $\mathrm{Sn} 3^{\text {viii }-P d 2-P d 7^{\text {viii }}}$ & $56.98(4)$ & $\mathrm{Sn} 2^{\mathrm{ix}}-\mathrm{Pd} 8-\mathrm{Pd} 5^{\mathrm{ix}}$ & $56.76(5)$ \\
\hline 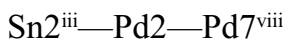 & $117.19(3)$ & $\mathrm{Pd} 3-\mathrm{Pd} 8-\mathrm{Pd} 5^{\mathrm{ix}}$ & $96.48(4)$ \\
\hline $\mathrm{Sn} 2-\mathrm{Pd} 2-\mathrm{Pd} 7^{\text {viii }}$ & $135.11(4)$ & $\mathrm{Sn} 4{ }^{\mathrm{vi}}-\mathrm{Pd} 8-\mathrm{Pd} 1$ & $61.35(9)$ \\
\hline $\mathrm{Pd} 5-\mathrm{Pd} 2-\mathrm{Pd} 7^{\text {viii }}$ & $121.22(7)$ & $\mathrm{Sn} 3-\mathrm{Pd} 8-\mathrm{Pd} 1$ & $146.27(5)$ \\
\hline $\mathrm{Pd} 55^{\mathrm{iii}}-\mathrm{Pd} 2-\mathrm{Pd} 7^{\mathrm{viii}}$ & $59.06(6)$ & $\mathrm{Sn} 2-\mathrm{Pd} 8-\mathrm{Pd} 1$ & $57.57(9)$ \\
\hline 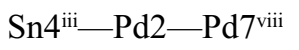 & $53.75(5)$ & $\mathrm{Sn} 5^{\mathrm{ii}}-\mathrm{Pd} 8-\mathrm{Pd} 1$ & $56.64(4)$ \\
\hline $\mathrm{Sn} 4-\mathrm{Pd} 2-\mathrm{Pd} 7^{\text {viii }}$ & $58.35(4)$ & $\mathrm{Pd} 9-\mathrm{Pd} 8-\mathrm{Pd} 1$ & $130.41(10)$ \\
\hline $\mathrm{Pd} 6-\mathrm{Pd} 2-\mathrm{Pd} 7^{\text {viii }}$ & $83.64(5)$ & $\mathrm{Pd} 4-\mathrm{Pd} 8-\mathrm{Pd} 1$ & $100.40(5)$ \\
\hline $\mathrm{Pd} 6{ }^{\mathrm{iii}}-\mathrm{Pd} 2-\mathrm{Pd} 7^{\mathrm{viii}}$ & $99.52(4)$ & $\mathrm{Sn} 2^{\mathrm{ix}}-\mathrm{Pd} 8-\mathrm{Pd} 1$ & $113.05(7)$ \\
\hline $\mathrm{Pd} 7^{\mathrm{vii}}-\mathrm{Pd} 2-\mathrm{Pd} 7^{\mathrm{viii}}$ & $86.36(9)$ & $\mathrm{Pd} 3-\mathrm{Pd} 8-\mathrm{Pd} 1$ & $57.15(5)$ \\
\hline $\mathrm{Sn} 2-\mathrm{Pd} 3-\mathrm{Sn} 2^{\mathrm{ix}}$ & $96.07(14)$ & $\mathrm{Pd} 5^{\mathrm{ix}}-\mathrm{Pd} 8-\mathrm{Pd} 1$ & $123.62(12)$ \\
\hline $\mathrm{Sn} 2-\mathrm{Pd} 3-\mathrm{Sn} 5^{\mathrm{x}}$ & $178.55(7)$ & $\mathrm{Sn} 4^{\mathrm{vi}}-\mathrm{Pd} 8-\mathrm{Pd} 4^{\mathrm{ii}}$ & $59.90(7)$ \\
\hline $\mathrm{Sn} 2^{\mathrm{ix}}-\mathrm{Pd} 3-\mathrm{Sn} 5^{\mathrm{x}}$ & $83.31(2)$ & $\mathrm{Sn} 3-\mathrm{Pd} 8-\mathrm{Pd} 4^{\mathrm{ii}}$ & $57.26(8)$ \\
\hline $\mathrm{Sn} 2-\mathrm{Pd} 3-\mathrm{Sn} 5^{\mathrm{i}}$ & $83.31(2)$ & $\mathrm{Sn} 2-\mathrm{Pd} 8-\mathrm{Pd} 4^{\mathrm{ii}}$ & $146.97(6)$ \\
\hline $\mathrm{Sn} 2^{\mathrm{ix}}-\mathrm{Pd} 3-\mathrm{Sn} 5^{\mathrm{i}}$ & $178.55(7)$ & 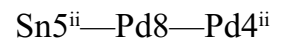 & $66.59(3)$ \\
\hline $\mathrm{Sn} 5^{\mathrm{x}}-\mathrm{Pd} 3-\mathrm{Sn} 5^{\mathrm{i}}$ & $97.34(13)$ & $\mathrm{Pd} 9-\mathrm{Pd} 8-\mathrm{Pd} 4^{\mathrm{ii}}$ & $130.97(10)$ \\
\hline $\mathrm{Sn} 2-\mathrm{Pd} 3-\mathrm{Pd} 1^{\mathrm{xi}}$ & $120.62(10)$ & $\mathrm{Pd} 4-\mathrm{Pd} 8-\mathrm{Pd} 4^{\mathrm{ii}}$ & $98.54(4)$ \\
\hline $\mathrm{Sn} 2^{\mathrm{ix}}-\mathrm{Pd} 3-\mathrm{Pd} 1^{\mathrm{xi}}$ & $59.12(10)$ & $\mathrm{Sn} 2^{\mathrm{ix}}-\mathrm{Pd} 8-\mathrm{Pd} 4^{\mathrm{ii}}$ & $113.73(7)$ \\
\hline
\end{tabular}




\begin{tabular}{|c|c|}
\hline $\mathrm{Sn} 5^{\mathrm{x}}-\mathrm{Pd} 3-\mathrm{Pd} 1^{\mathrm{xi}}$ & $57.95(4)$ \\
\hline $\mathrm{Sn} 5^{\mathrm{i}}-\mathrm{Pd} 3-\mathrm{Pd} 1^{\mathrm{xi}}$ & $122.31(15)$ \\
\hline $\mathrm{Sn} 2-\mathrm{Pd} 3-\mathrm{Pd} 1$ & $59.12(10)$ \\
\hline $\mathrm{Sn} 2^{\mathrm{ix}}-\mathrm{Pd} 3-\mathrm{Pd} 1$ & $120.62(10)$ \\
\hline $\mathrm{Sn} 5^{\mathrm{x}}-\mathrm{Pd} 3-\mathrm{Pd} 1$ & $122.32(15)$ \\
\hline 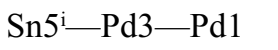 & $57.95(4)$ \\
\hline $\mathrm{Pd} 1^{\mathrm{xi}}-\mathrm{Pd} 3-\mathrm{Pd} 1$ & $179.7(2)$ \\
\hline $\mathrm{Sn} 2-\mathrm{Pd} 3-\mathrm{Pd} 7^{\mathrm{x}}$ & $121.64(4)$ \\
\hline $\mathrm{Sn} 2^{\mathrm{ix}}-\mathrm{Pd} 3-\mathrm{Pd} 7^{\mathrm{x}}$ & $122.05(4)$ \\
\hline $\mathrm{Sn} 5^{\mathrm{x}}-\mathrm{Pd} 3-\mathrm{Pd} 7^{\mathrm{x}}$ & $57.85(8)$ \\
\hline $\mathrm{Sn} 5^{\mathrm{i}}-\mathrm{Pd} 3-\mathrm{Pd} 7^{\mathrm{x}}$ & $59.33(8)$ \\
\hline$P d 1^{x i}-P d 3-P d 7^{x}$ & $63.84(10)$ \\
\hline $\mathrm{Pd} 1-\mathrm{Pd} 3-\mathrm{Pd} 7^{\mathrm{x}}$ & $116.45(11)$ \\
\hline $\mathrm{Sn} 2-\mathrm{Pd} 3-\mathrm{Pd} 7^{\mathrm{i}}$ & $122.05(4)$ \\
\hline $\mathrm{Sn} 2^{\mathrm{ix}}-\mathrm{Pd} 3-\mathrm{Pd} 7^{\mathrm{i}}$ & $121.64(4)$ \\
\hline $\mathrm{Sn} 5^{\mathrm{x}}-\mathrm{Pd} 3-\mathrm{Pd} 7^{\mathrm{i}}$ & $59.33(8)$ \\
\hline 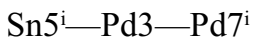 & $57.85(8)$ \\
\hline $\mathrm{Pd} 1^{\mathrm{xi}}-\mathrm{Pd} 3-\mathrm{Pd} 7^{\mathrm{i}}$ & $116.45(11)$ \\
\hline $\mathrm{Pd} 1-\mathrm{Pd} 3-\mathrm{Pd} 7^{\mathrm{i}}$ & $63.84(10)$ \\
\hline $\mathrm{Pd} 7^{\mathrm{x}}-\mathrm{Pd} 3-\mathrm{Pd} 7^{\mathrm{i}}$ & $75.79(12)$ \\
\hline $\mathrm{Sn} 2-\mathrm{Pd} 3-\mathrm{Pd} 8^{\mathrm{ix}}$ & $59.72(8)$ \\
\hline $\mathrm{Sn} 2^{\mathrm{ix}}-\mathrm{Pd} 3-\mathrm{Pd} 8^{\mathrm{ix}}$ & $57.22(8)$ \\
\hline $\mathrm{Sn} 5^{\mathrm{x}}-\mathrm{Pd} 3-\mathrm{Pd} 8^{\mathrm{ix}}$ & $118.91(4)$ \\
\hline $\mathrm{Sn} 5^{\mathrm{i}}-\mathrm{Pd} 3-\mathrm{Pd} 8^{\mathrm{ix}}$ & $123.33(4)$ \\
\hline $\mathrm{Pd} 1^{\mathrm{xi}}-\mathrm{Pd} 3-\mathrm{Pd} 8^{\mathrm{ix}}$ & $61.85(5)$ \\
\hline $\mathrm{Pd} 1-\mathrm{Pd} 3-\mathrm{Pd} 8^{\mathrm{ix}}$ & $117.85(15)$ \\
\hline $\mathrm{Pd} 7^{\mathrm{x}}-\mathrm{Pd} 3-\mathrm{Pd} 8^{\mathrm{ix}}$ & $103.57(3)$ \\
\hline $\mathrm{Pd} 7^{\mathrm{i}}-\mathrm{Pd} 3-\mathrm{Pd} 8^{\mathrm{ix}}$ & $178.21(6)$ \\
\hline $\mathrm{Sn} 2-\mathrm{Pd} 3-\mathrm{Pd} 8$ & $57.22(8)$ \\
\hline $\mathrm{Sn} 2^{2 \mathrm{x}}-\mathrm{Pd} 3-\mathrm{Pd} 8$ & $59.72(8)$ \\
\hline $\mathrm{Sn} 5^{\mathrm{x}}-\mathrm{Pd} 3-\mathrm{Pd} 8$ & $123.32(4)$ \\
\hline $\mathrm{Sn} 5^{\mathrm{i}}-\mathrm{Pd} 3-\mathrm{Pd} 8$ & $118.91(4)$ \\
\hline $\mathrm{Pd} 1{ }^{\mathrm{xi}}-\mathrm{Pd} 3-\mathrm{Pd} 8$ & $117.85(15)$ \\
\hline $\mathrm{Pd} 1-\mathrm{Pd} 3-\mathrm{Pd} 8$ & $61.85(5)$ \\
\hline $\mathrm{Pd} 77^{\mathrm{x}}-\mathrm{Pd} 3-\mathrm{Pd} 8$ & $178.21(6)$ \\
\hline $\mathrm{Pd} 7^{\mathrm{i}}-\mathrm{Pd} 3-\mathrm{Pd} 8$ & $103.57(3)$ \\
\hline $\mathrm{Pd} 88^{\mathrm{ix}}-\mathrm{Pd} 3-\mathrm{Pd} 8$ & $77.12(11)$ \\
\hline $\mathrm{Sn} 2-\mathrm{Pd} 3-\mathrm{Sn} 4^{\mathrm{vi}}$ & $101.81(8)$ \\
\hline $\mathrm{Sn} 2^{\mathrm{ix}}-\mathrm{Pd} 3-\mathrm{Sn} 4^{\mathrm{vi}}$ & $77.90(6)$ \\
\hline $\mathrm{Sn} 5^{\mathrm{x}}-\mathrm{Pd} 3-\mathrm{Sn} 4^{\mathrm{vi}}$ & $79.35(6)$ \\
\hline $\mathrm{Sn} 5^{\mathrm{i}}-\mathrm{Pd} 3-\mathrm{Sn} 4^{\mathrm{vi}}$ & $100.92(7)$ \\
\hline $\mathrm{Pd} 1^{\mathrm{xi}}-\mathrm{Pd} 3-\mathrm{Sn} 4^{\mathrm{vi}}$ & $120.39(7)$ \\
\hline $\mathrm{Pd} 1-\mathrm{Pd} 3-\mathrm{Sn} 4^{\mathrm{vi}}$ & $59.61(7)$ \\
\hline $\mathrm{Pd} 7^{\mathrm{x}}-\mathrm{Pd} 3-\mathrm{Sn} 4^{\mathrm{vi}}$ & $126.43(14)$ \\
\hline $\mathrm{Pd} 7^{\mathrm{i}}-\mathrm{Pd} 3-\mathrm{Sn} 4^{\mathrm{vi}}$ & $53.97(4)$ \\
\hline $\mathrm{Pd} 8^{\mathrm{ix}}-\mathrm{Pd} 3-\mathrm{Sn} 4^{\mathrm{vi}}$ & $126.04(13)$ \\
\hline $\mathrm{Pd} 8-\mathrm{Pd} 3-\mathrm{Sn} 4^{\mathrm{vi}}$ & $53.55(4)$ \\
\hline $\mathrm{Sn} 2-\mathrm{Pd} 3-\mathrm{Sn} 4^{\mathrm{xii}}$ & $77.90(6)$ \\
\hline
\end{tabular}

\begin{tabular}{|c|c|}
\hline $\mathrm{Pd} 3-\mathrm{Pd} 8-\mathrm{Pd} 44^{\mathrm{ii}}$ & $123.68(10)$ \\
\hline $\mathrm{Pd} 5^{\mathrm{ix}}-\mathrm{Pd} 8-\mathrm{Pd} 4^{\mathrm{ii}}$ & $57.83(5)$ \\
\hline $\mathrm{Pd} 1-\mathrm{Pd} 8-\mathrm{Pd} 4^{\mathrm{ii}}$ & $94.05(4)$ \\
\hline $\mathrm{Pd} 7-\mathrm{Pd} 9-\mathrm{Pd} 7^{\mathrm{ix}}$ & $80.14(9)$ \\
\hline $\mathrm{Pd} 7-\mathrm{Pd} 9-\mathrm{Pd} 6^{\mathrm{ix}}$ & $80.90(7)$ \\
\hline $\mathrm{Pd} 7^{\mathrm{ix}}-\mathrm{Pd} 9-\mathrm{Pd} 6^{\mathrm{ix}}$ & $99.86(4)$ \\
\hline $\mathrm{Pd} 7-\mathrm{Pd} 9-\mathrm{Pd} 6$ & $99.87(4)$ \\
\hline $\mathrm{Pd} 77^{\mathrm{ix}}-\mathrm{Pd} 9-\mathrm{Pd} 6$ & $80.90(7)$ \\
\hline $\mathrm{Pd} 66^{\mathrm{ix}}-\mathrm{Pd} 9-\mathrm{Pd} 6$ & $179.02(12)$ \\
\hline $\mathrm{Pd} 7-\mathrm{Pd} 9-\mathrm{Pd} 4$ & $61.16(6)$ \\
\hline $\mathrm{Pd} 77^{\mathrm{ix}}-\mathrm{Pd} 9-\mathrm{Pd} 4$ & $116.98(7)$ \\
\hline $\mathrm{Pd} 66^{\mathrm{ix}}-\mathrm{Pd} 9-\mathrm{Pd} 4$ & $118.52(8)$ \\
\hline $\mathrm{Pd} 6-\mathrm{Pd} 9-\mathrm{Pd} 4$ & $61.50(8)$ \\
\hline $\mathrm{Pd} 7-\mathrm{Pd} 9-\mathrm{Pd} 4^{\mathrm{ix}}$ & $116.98(7)$ \\
\hline $\mathrm{Pd} 7^{\mathrm{ix}}-\mathrm{Pd} 9-\mathrm{Pd} 4^{\mathrm{ix}}$ & $61.16(6)$ \\
\hline $\mathrm{Pd} 6^{\mathrm{ix}}-\mathrm{Pd} 9-\mathrm{Pd} 4^{\mathrm{ix}}$ & $61.50(8)$ \\
\hline $\mathrm{Pd} 6-\mathrm{Pd} 9-\mathrm{Pd} 4^{\mathrm{ix}}$ & $118.52(8)$ \\
\hline $\mathrm{Pd} 4-\mathrm{Pd} 9-\mathrm{Pd} 4^{\mathrm{ix}}$ & $177.85(12)$ \\
\hline $\mathrm{Pd} 7-\mathrm{Pd} 9-\mathrm{Pd} 8^{\mathrm{ix}}$ & $178.02(4)$ \\
\hline $\mathrm{Pd} 7^{\mathrm{ix}}-\mathrm{Pd} 9-\mathrm{Pd} 8^{\mathrm{ix}}$ & $98.94(3)$ \\
\hline $\mathrm{Pd} 6^{\mathrm{ix}}-\mathrm{Pd} 9-\mathrm{Pd} 8^{\mathrm{ix}}$ & $97.57(4)$ \\
\hline $\mathrm{Pd} 6-\mathrm{Pd} 9-\mathrm{Pd} 8^{\mathrm{ix}}$ & $81.68(7)$ \\
\hline $\mathrm{Pd} 4-\mathrm{Pd} 9-\mathrm{Pd} 8^{\mathrm{ix}}$ & $120.78(8)$ \\
\hline $\mathrm{Pd} 4^{\mathrm{ix}}-\mathrm{Pd} 9-\mathrm{Pd} 8^{\mathrm{ix}}$ & $61.08(5)$ \\
\hline $\mathrm{Pd} 7-\mathrm{Pd} 9-\mathrm{Pd} 8$ & $98.94(3)$ \\
\hline $\mathrm{Pd} 7^{\mathrm{ix}}-\mathrm{Pd} 9-\mathrm{Pd} 8$ & $178.02(4)$ \\
\hline $\mathrm{Pd} 6^{\mathrm{ix}}-\mathrm{Pd} 9-\mathrm{Pd} 8$ & $81.69(7)$ \\
\hline $\mathrm{Pd} 6-\mathrm{Pd} 9-\mathrm{Pd} 8$ & $97.57(4)$ \\
\hline $\mathrm{Pd} 4-\mathrm{Pd} 9-\mathrm{Pd} 8$ & $61.08(5)$ \\
\hline $\mathrm{Pd} 4^{\mathrm{ix}}-\mathrm{Pd} 9-\mathrm{Pd} 8$ & $120.78(8)$ \\
\hline $\mathrm{Pd} 8^{\mathrm{ix}}-\mathrm{Pd} 9-\mathrm{Pd} 8$ & $82.03(9)$ \\
\hline $\mathrm{Pd} 7-\mathrm{Pd} 9-\mathrm{Sn} 2$ & $127.01(2)$ \\
\hline $\mathrm{Pd} 7^{\mathrm{ix}}-\mathrm{Pd} 9-\mathrm{Sn} 2$ & $126.63(6)$ \\
\hline $\mathrm{Pd} 6^{\mathrm{ix}}-\mathrm{Pd} 9-\mathrm{Sn} 2$ & $126.54(8)$ \\
\hline $\mathrm{Pd} 6-\mathrm{Pd} 9-\mathrm{Sn} 2$ & $52.49(6)$ \\
\hline $\mathrm{Pd} 4-\mathrm{Pd} 9-\mathrm{Sn} 2$ & $65.85(6)$ \\
\hline $\mathrm{Pd} 4{ }^{\mathrm{x}}-\mathrm{Pd} 9-\mathrm{Sn} 2$ & $116.00(6)$ \\
\hline $\mathrm{Pd} 8^{\mathrm{ix}}-\mathrm{Pd} 9-\mathrm{Sn} 2$ & $54.93(4)$ \\
\hline $\mathrm{Pd} 8-\mathrm{Pd} 9-\mathrm{Sn} 2$ & $52.61(5)$ \\
\hline $\mathrm{Pd} 7-\mathrm{Pd} 9-\mathrm{Sn} 2^{\mathrm{ix}}$ & $126.63(6)$ \\
\hline $\mathrm{Pd} 7^{\mathrm{ix}}-\mathrm{Pd} 9-\mathrm{Sn} 2^{\mathrm{ix}}$ & $127.01(2)$ \\
\hline $\mathrm{Pd} 6^{\mathrm{ix}}-\mathrm{Pd} 9-\mathrm{Sn} 2^{\mathrm{ix}}$ & $52.49(6)$ \\
\hline $\mathrm{Pd} 6-\mathrm{Pd} 9-\mathrm{Sn} 2^{\mathrm{ix}}$ & $126.54(8)$ \\
\hline $\mathrm{Pd} 4-\mathrm{Pd} 9-\mathrm{Sn} 2^{\mathrm{ix}}$ & $116.00(6)$ \\
\hline $\mathrm{Pd} 4^{\mathrm{ix}}-\mathrm{Pd} 9-\mathrm{Sn} 2^{\mathrm{ix}}$ & $65.85(6)$ \\
\hline $\mathrm{Pd} 8^{\mathrm{ix}}-\mathrm{Pd} 9-\mathrm{Sn} 2^{\mathrm{ix}}$ & $52.61(5)$ \\
\hline $\mathrm{Pd} 8-\mathrm{Pd} 9-\mathrm{Sn} 2^{\mathrm{ix}}$ & $54.93(4)$ \\
\hline $\mathrm{Sn} 2-\mathrm{Pd} 9-\mathrm{Sn} 2^{\mathrm{ix}}$ & $76.90(7)$ \\
\hline
\end{tabular}




\begin{tabular}{|c|c|}
\hline $\mathrm{Sn} 2^{\mathrm{ix}}-\mathrm{Pd} 3-\mathrm{Sn} 4^{\mathrm{xii}}$ & $101.81(8)$ \\
\hline $\mathrm{Sn} 5^{\mathrm{x}}-\mathrm{Pd} 3-\mathrm{Sn} 4^{\mathrm{xii}}$ & $100.92(7)$ \\
\hline 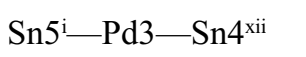 & $79.35(6)$ \\
\hline $\mathrm{Pd} 1^{\mathrm{xi}}-\mathrm{Pd} 3-\mathrm{Sn} 4^{\mathrm{xii}}$ & $59.61(7)$ \\
\hline $\mathrm{Pd} 1-\mathrm{Pd} 3-\mathrm{Sn} 4^{\mathrm{xii}}$ & $120.39(7)$ \\
\hline $\mathrm{Pd} 7^{\mathrm{x}}-\mathrm{Pd} 3-\mathrm{Sn} 4^{\mathrm{xii}}$ & $53.97(4)$ \\
\hline $\mathrm{Pd} 7^{\mathrm{i}}-\mathrm{Pd} 3-\mathrm{Sn} 4^{\mathrm{xii}}$ & $126.43(14)$ \\
\hline $\mathrm{Pd} 8^{\mathrm{ix}}-\mathrm{Pd} 3-\mathrm{Sn} 4^{\mathrm{xii}}$ & $53.56(4)$ \\
\hline $\mathrm{Pd} 8-\mathrm{Pd} 3-\mathrm{Sn} 4^{\mathrm{xii}}$ & 126.04 \\
\hline $\mathrm{Sn} 4^{\mathrm{vi}}-\mathrm{Pd} 3-\mathrm{Sn} 4^{\mathrm{xii}}$ & $179.59(18)$ \\
\hline $\mathrm{Sn} 3^{\mathrm{viii}}-\mathrm{Pd} 4-\mathrm{Sn} 5^{\mathrm{ii}}$ & $84.03(6)$ \\
\hline 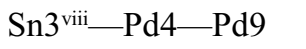 & $119.64(12)$ \\
\hline $\mathrm{Sn} 55^{\mathrm{ii}}-\mathrm{Pd} 4-\mathrm{Pd} 9$ & $119.22(7)$ \\
\hline 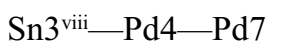 & $137.40(9)$ \\
\hline $\mathrm{Sn} 5^{\mathrm{ii}}-\mathrm{Pd} 4-\mathrm{Pd} 7$ & $136.41(14)$ \\
\hline $\mathrm{Pd} 9-\mathrm{Pd} 4-\mathrm{Pd} 7$ & $59.22(5)$ \\
\hline 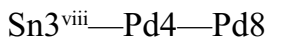 & $119.74(10)$ \\
\hline 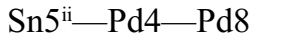 & $59.96(5)$ \\
\hline $\mathrm{Pd} 9-\mathrm{Pd} 4-\mathrm{Pd} 8$ & $59.85(5)$ \\
\hline $\mathrm{Pd} 7-\mathrm{Pd} 4-\mathrm{Pd} 8$ & $96.87(7)$ \\
\hline 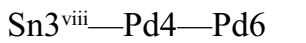 & $61.11(8)$ \\
\hline $\mathrm{Sn} 5^{\mathrm{ii}}-\mathrm{Pd} 4-\mathrm{Pd} 6$ & $119.88(11)$ \\
\hline $\mathrm{Pd} 9-\mathrm{Pd} 4-\mathrm{Pd} 6$ & $59.12(4)$ \\
\hline $\mathrm{Pd} 7-\mathrm{Pd} 4-\mathrm{Pd} 6$ & $97.00(5)$ \\
\hline $\mathrm{Pd} 8-\mathrm{Pd} 4-\mathrm{Pd} 6$ & $95.27(6)$ \\
\hline $\mathrm{Sn} 3^{\mathrm{viii}}-\mathrm{Pd} 4-\mathrm{Sn} 4^{\mathrm{ii}}$ & $103.82(5)$ \\
\hline $\mathrm{Sn} 5^{\mathrm{ii}}-\mathrm{Pd} 4-\mathrm{Sn} 4^{\mathrm{ii}}$ & $103.15(8)$ \\
\hline $\mathrm{Pd} 9-\mathrm{Pd} 4-\mathrm{Sn} 4^{\mathrm{ii}}$ & $120.30(9)$ \\
\hline $\mathrm{Pd} 7-\mathrm{Pd} 4-\mathrm{Sn} 4^{\mathrm{ii}}$ & $61.08(6)$ \\
\hline $\mathrm{Pd} 8-\mathrm{Pd} 4-\mathrm{Sn} 4^{\mathrm{ii}}$ & $128.95(14)$ \\
\hline $\mathrm{Pd} 6-\mathrm{Pd} 4-\mathrm{Sn} 4^{\mathrm{ii}}$ & $130.44(9)$ \\
\hline $\mathrm{Sn} 3^{\text {viii }-P d 4-P d 5^{\text {xiii }}}$ & $59.65(9)$ \\
\hline $\mathrm{Sn} 5^{\mathrm{ii}}-\mathrm{Pd} 4-\mathrm{Pd} 5^{\mathrm{xiii}}$ & $59.32(6)$ \\
\hline $\mathrm{Pd} 9-\mathrm{Pd} 4-\mathrm{Pd} 5^{\mathrm{xiii}}$ & $178.23(11)$ \\
\hline $\mathrm{Pd} 7-\mathrm{Pd} 4-\mathrm{Pd} 5^{\mathrm{xiii}}$ & $122.48(10)$ \\
\hline $\mathrm{Pd} 8-\mathrm{Pd} 4-\mathrm{Pd} 5^{\mathrm{xiii}}$ & $118.85(9)$ \\
\hline $\mathrm{Pd} 6-\mathrm{Pd} 4-\mathrm{Pd} 5^{\mathrm{xiii}}$ & $120.40(14)$ \\
\hline $\mathrm{Sn} 4^{\mathrm{ii}}-\mathrm{Pd} 4-\mathrm{Pd} 5^{\mathrm{xiii}}$ & $61.40(6)$ \\
\hline 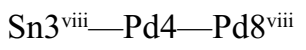 & $56.49(6)$ \\
\hline $\mathrm{Sn} 5^{\mathrm{ii}}-\mathrm{Pd} 4-\mathrm{Pd} 8^{\mathrm{viii}}$ & $118.66(5)$ \\
\hline $\mathrm{Pd} 9-\mathrm{Pd} 4-\mathrm{Pd} 8^{\text {viii }}$ & $120.82(8)$ \\
\hline $\mathrm{Pd} 7-\mathrm{Pd} 4-\mathrm{Pd} 8^{\text {viii }}$ & $86.42(5)$ \\
\hline $\mathrm{Pd} 8-\mathrm{Pd} 4-\mathrm{Pd} 8^{\text {viii }}$ & $176.19(13)$ \\
\hline $\mathrm{Pd} 6-\mathrm{Pd} 4-\mathrm{Pd} 8^{\text {viii }}$ & $82.38(9)$ \\
\hline $\mathrm{Sn} 4^{\mathrm{ii}}-\mathrm{Pd} 4-\mathrm{Pd} 8^{\text {viii }}$ & $54.45(5)$ \\
\hline $\mathrm{Pd} 5^{\mathrm{xiii}}-\mathrm{Pd} 4-\mathrm{Pd} 8^{\mathrm{viii}}$ & $60.38(7)$ \\
\hline $\mathrm{Sn} 3^{\mathrm{viii}}-\mathrm{Pd} 4-\mathrm{Pd} 6^{\mathrm{ii}}$ & $117.94(5)$ \\
\hline $\mathrm{Sn} 5^{\mathrm{ii}}-\mathrm{Pd} 4-\mathrm{Pd} 6^{\mathrm{ii}}$ & $56.01(6)$ \\
\hline
\end{tabular}

\begin{tabular}{|c|c|}
\hline $\mathrm{Pd} 6-\mathrm{Sn} 2-\mathrm{Pd} 8$ & $100.35(4)$ \\
\hline $\mathrm{Pd} 6-\mathrm{Sn} 2-\mathrm{Pd} 3$ & $143.25(7)$ \\
\hline $\mathrm{Pd} 8-\mathrm{Sn} 2-\mathrm{Pd} 3$ & $64.99(7)$ \\
\hline $\mathrm{Pd} 6-\mathrm{Sn} 2-\mathrm{Pd} 2$ & $66.12(6)$ \\
\hline $\mathrm{Pd} 8-\mathrm{Sn} 2-\mathrm{Pd} 2$ & $144.55(4)$ \\
\hline $\mathrm{Pd} 3-\mathrm{Sn} 2-\mathrm{Pd} 2$ & $144.15(7)$ \\
\hline $\mathrm{Pd} 6-\mathrm{Sn} 2-\mathrm{Pd} 5$ & $65.14(8)$ \\
\hline $\mathrm{Pd} 8-\mathrm{Sn} 2-\mathrm{Pd} 5$ & $144.00(6)$ \\
\hline $\mathrm{Pd} 3-\mathrm{Sn} 2-\mathrm{Pd} 5$ & $106.02(4)$ \\
\hline $\mathrm{Pd} 2-\mathrm{Sn} 2-\mathrm{Pd} 5$ & $62.27(5)$ \\
\hline $\mathrm{Pd} 6-\mathrm{Sn} 2-\mathrm{Pd} 1$ & $145.28(4)$ \\
\hline $\mathrm{Pd} 8-\mathrm{Sn} 2-\mathrm{Pd} 1$ & $65.54(7)$ \\
\hline $\mathrm{Pd} 3-\mathrm{Sn} 2-\mathrm{Pd} 1$ & $61.87(6)$ \\
\hline $\mathrm{Pd} 2-\mathrm{Sn} 2-\mathrm{Pd} 1$ & $106.14(5)$ \\
\hline $\mathrm{Pd} 5-\mathrm{Sn} 2-\mathrm{Pd} 1$ & $143.76(8)$ \\
\hline $\mathrm{Pd} 6-\mathrm{Sn} 2-\mathrm{Pd} 8^{\mathrm{ix}}$ & $81.77(6)$ \\
\hline $\mathrm{Pd} 8-\mathrm{Sn} 2-\mathrm{Pd} 8^{\mathrm{ix}}$ & $82.40(5)$ \\
\hline $\mathrm{Pd} 3-\mathrm{Sn} 2-\mathrm{Pd} 8^{\mathrm{ix}}$ & $63.56(7)$ \\
\hline $\mathrm{Pd} 2-\mathrm{Sn} 2-\mathrm{Pd} 8^{\mathrm{ix}}$ & $124.61(8)$ \\
\hline $\mathrm{Pd} 5-\mathrm{Sn} 2-\mathrm{Pd} 8^{\mathrm{ix}}$ & $63.55(6)$ \\
\hline $\mathrm{Pd} 1-\mathrm{Sn} 2-\mathrm{Pd} 8^{\mathrm{ix}}$ & $124.30(10)$ \\
\hline $\mathrm{Pd} 6-\mathrm{Sn} 2-\mathrm{Pd} 9$ & $53.86(4)$ \\
\hline $\mathrm{Pd} 8-\mathrm{Sn} 2-\mathrm{Pd} 9$ & $54.43(3)$ \\
\hline $\mathrm{Pd} 3-\mathrm{Sn} 2-\mathrm{Pd} 9$ & $93.51(6)$ \\
\hline $\mathrm{Pd} 2-\mathrm{Sn} 2-\mathrm{Pd} 9$ & $119.84(6)$ \\
\hline $\mathrm{Pd} 5-\mathrm{Sn} 2-\mathrm{Pd} 9$ & $93.67(7)$ \\
\hline $\mathrm{Pd} 1-\mathrm{Sn} 2-\mathrm{Pd} 9$ & $119.88(7)$ \\
\hline $\mathrm{Pd} 8^{\mathrm{ix}}-\mathrm{Sn} 2-\mathrm{Pd} 9$ & $53.72(3)$ \\
\hline $\mathrm{Pd} 6-\mathrm{Sn} 2-\mathrm{Sn} 2^{\mathrm{iii}}$ & $110.29(4)$ \\
\hline $\mathrm{Pd} 8-\mathrm{Sn} 2-\mathrm{Sn} 2^{\mathrm{iii}}$ & $109.49(3)$ \\
\hline $\mathrm{Pd} 3-\mathrm{Sn} 2-\mathrm{Sn} 2^{\mathrm{iii}}$ & $106.41(6)$ \\
\hline $\mathrm{Pd} 2-\mathrm{Sn} 2-\mathrm{Sn} 2^{\mathrm{iii}}$ & $53.05(4)$ \\
\hline $\mathrm{Pd} 5-\mathrm{Sn} 2-\mathrm{Sn} 2^{\mathrm{iii}}$ & $106.48(6)$ \\
\hline $\mathrm{Pd} 1-\mathrm{Sn} 2-\mathrm{Sn} 2^{\mathrm{iii}}$ & $53.09(6)$ \\
\hline $\mathrm{Pd} 8^{\mathrm{ix}}-\mathrm{Sn} 2-\mathrm{Sn} 2^{\mathrm{iii}}$ & $160.33(5)$ \\
\hline $\mathrm{Pd} 9-\mathrm{Sn} 2-\mathrm{Sn} 2^{\mathrm{iii}}$ & $145.95(4)$ \\
\hline $\mathrm{Pd} 8-\mathrm{Sn} 3-\mathrm{Pd} 2^{\mathrm{xvi}}$ & $148.03(5)$ \\
\hline $\mathrm{Pd} 8-\mathrm{Sn} 3-\mathrm{Pd} 4^{\mathrm{ii}}$ & $66.25(7)$ \\
\hline $\mathrm{Pd} 2^{\mathrm{xvi}}-\mathrm{Sn} 3-\mathrm{Pd} 4^{\mathrm{ii}}$ & $108.48(6)$ \\
\hline $\mathrm{Pd} 8-\mathrm{Sn} 3-\mathrm{Pd} 7$ & $101.00(4)$ \\
\hline $\mathrm{Pd} 2^{\mathrm{xvi}}-\mathrm{Sn} 3-\mathrm{Pd} 7$ & $66.65(7)$ \\
\hline $\mathrm{Pd} 4{ }^{\mathrm{ii}}-\mathrm{Sn} 3-\mathrm{Pd} 7$ & $148.98(6)$ \\
\hline $\mathrm{Pd} 8-\mathrm{Sn} 3-\mathrm{Pd} 5^{\mathrm{ix}}$ & $64.58(6)$ \\
\hline $\mathrm{Pd} 2^{\mathrm{xvi}}-\mathrm{Sn} 3-\mathrm{Pd} 5^{\mathrm{ix}}$ & $143.39(7)$ \\
\hline $\mathrm{Pd} 4^{\mathrm{ii}}-\mathrm{Sn} 3-\mathrm{Pd} 5^{\mathrm{ix}}$ & $62.39(7)$ \\
\hline $\mathrm{Pd} 7-\mathrm{Sn} 3-\mathrm{Pd} 5^{\mathrm{ix}}$ & $139.85(6)$ \\
\hline $\mathrm{Pd} 8-\mathrm{Sn} 3-\mathrm{Pd} 5^{\mathrm{xvi}}$ & $140.76(6)$ \\
\hline $\mathrm{Pd} 2^{\mathrm{xvi}}-\mathrm{Sn} 3-\mathrm{Pd} 5^{\mathrm{xvi}}$ & $61.69(6)$ \\
\hline
\end{tabular}




\begin{tabular}{|c|c|}
\hline $\mathrm{Pd} 9-\mathrm{Pd} 4-\mathrm{Pd} 6^{\mathrm{ii}}$ & $121.00(11)$ \\
\hline $\mathrm{Pd} 7-\mathrm{Pd} 4-\mathrm{Pd} 6^{\mathrm{ii}}$ & $86.30(10)$ \\
\hline $\mathrm{Pd} 8-\mathrm{Pd} 4-\mathrm{Pd} 6^{\mathrm{ii}}$ & $81.61(8)$ \\
\hline $\mathrm{Pd} 6-\mathrm{Pd} 4-\mathrm{Pd} 6^{\mathrm{ii}}$ & $175.73(7)$ \\
\hline $\mathrm{Sn} 4^{4 i}-\mathrm{Pd} 4-\mathrm{Pd} 6^{\mathrm{ii}}$ & $53.63(7)$ \\
\hline $\mathrm{Pd} 5^{\mathrm{xiii}}-\mathrm{Pd} 4-\mathrm{Pd} 6^{\mathrm{ii}}$ & $59.33(9)$ \\
\hline $\mathrm{Pd} 8^{\mathrm{viii}}-\mathrm{Pd} 4-\mathrm{Pd} 6^{\mathrm{ii}}$ & $100.58(5)$ \\
\hline 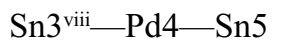 & $84.60(8)$ \\
\hline $\mathrm{Sn} 55^{\mathrm{ii}}-\mathrm{Pd} 4-\mathrm{Sn} 5$ & $168.62(12)$ \\
\hline $\mathrm{Pd} 9-\mathrm{Pd} 4-\mathrm{Sn} 5$ & $67.09(5)$ \\
\hline $\mathrm{Pd} 7-\mathrm{Pd} 4-\mathrm{Sn} 5$ & $54.59(5)$ \\
\hline $\mathrm{Pd} 8-\mathrm{Pd} 4-\mathrm{Sn} 5$ & $126.91(3)$ \\
\hline $\mathrm{Pd} 6-\mathrm{Pd} 4-\mathrm{Sn} 5$ & $53.61(7)$ \\
\hline $\mathrm{Sn} 44^{\mathrm{ii}}-\mathrm{Pd} 4-\mathrm{Sn} 5$ & $79.51(5)$ \\
\hline $\mathrm{Pd} 5^{\mathrm{xiii}}-\mathrm{Pd} 4-\mathrm{Sn} 5$ & $114.16(9)$ \\
\hline 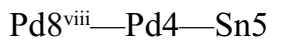 & $53.79(4)$ \\
\hline $\mathrm{Pd} 66^{\mathrm{ii}}-\mathrm{Pd} 4-\mathrm{Sn} 5$ & $130.65(9)$ \\
\hline $\mathrm{Sn} 2-\mathrm{Pd} 5-\mathrm{Sn} 5^{\mathrm{xii}}$ & $178.71(10)$ \\
\hline $\mathrm{Sn} 2-\mathrm{Pd} 5-\mathrm{Sn} 3^{\mathrm{ix}}$ & $96.39(11)$ \\
\hline $\mathrm{Sn} 5^{\mathrm{xii}}-\mathrm{Pd} 5-\mathrm{Sn} 3^{\mathrm{ix}}$ & $82.57(4)$ \\
\hline $\mathrm{Sn} 2-\mathrm{Pd} 5-\mathrm{Sn} 3^{\mathrm{vii}}$ & $83.37(4)$ \\
\hline $\mathrm{Sn} 5^{\mathrm{xii}}-\mathrm{Pd} 5-\mathrm{Sn} 3^{\mathrm{vii}}$ & $97.68(11)$ \\
\hline $\mathrm{Sn} 3^{\mathrm{ix}}-\mathrm{Pd} 5-\mathrm{Sn} 3^{\mathrm{vii}}$ & $179.44(10)$ \\
\hline $\mathrm{Sn} 2-\mathrm{Pd} 5-\mathrm{Pd} 2$ & $58.84(6)$ \\
\hline 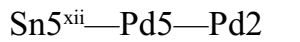 & $122.37(9)$ \\
\hline $\mathrm{Sn} 3^{\mathrm{ix}}-\mathrm{Pd} 5-\mathrm{Pd} 2$ & $121.51(11)$ \\
\hline 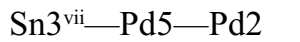 & $57.93(4)$ \\
\hline $\mathrm{Sn} 2-\mathrm{Pd} 5-\mathrm{Pd} 4^{\mathrm{xiv}}$ & $120.49(9)$ \\
\hline $\mathrm{Sn} 5^{\mathrm{xii}}-\mathrm{Pd} 5-\mathrm{Pd} 4^{\mathrm{xiv}}$ & $58.30(5)$ \\
\hline $\mathrm{Sn} 3^{\mathrm{ix}}-\mathrm{Pd} 5-\mathrm{Pd} 4^{\mathrm{xiv}}$ & $57.96(9)$ \\
\hline 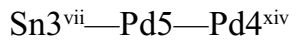 & $122.59(15)$ \\
\hline $\mathrm{Pd} 2-\mathrm{Pd} 5-\mathrm{Pd} 4^{\mathrm{xiv}}$ & $179.22(17)$ \\
\hline $\mathrm{Sn} 2-\mathrm{Pd} 5-\mathrm{Pd} 7^{\mathrm{vii}}$ & $121.49(5)$ \\
\hline $\mathrm{Sn} 5^{\mathrm{xii}}-\mathrm{Pd} 5-\mathrm{Pd} 7^{\mathrm{vii}}$ & $59.78(6)$ \\
\hline $\mathrm{Sn} 3^{\mathrm{ix}}-\mathrm{Pd} 5-\mathrm{Pd} 7^{\mathrm{vii}}$ & $121.99(7)$ \\
\hline $\mathrm{Sn} 3^{\mathrm{vii}}-\mathrm{Pd} 5-\mathrm{Pd} 7^{\mathrm{vii}}$ & $57.81(7)$ \\
\hline $\mathrm{Pd} 2-\mathrm{Pd} 5-\mathrm{Pd} 7^{\mathrm{vii}}$ & $63.48(6)$ \\
\hline $\mathrm{Pd} 4^{\mathrm{xiv}}-\mathrm{Pd} 5-\mathrm{Pd} 7^{\mathrm{vii}}$ & $117.25(10)$ \\
\hline $\mathrm{Sn} 2-\mathrm{Pd} 5-\mathrm{Sn} 4^{\mathrm{xii}}$ & $78.94(6)$ \\
\hline $\mathrm{Sn} 5^{\mathrm{xii}}-\mathrm{Pd} 5-\mathrm{Sn} 4^{\mathrm{xii}}$ & $100.48(7)$ \\
\hline $\mathrm{Sn} 3^{\mathrm{ix}}-\mathrm{Pd} 5-\mathrm{Sn} 4^{\mathrm{xii}}$ & $100.64(6)$ \\
\hline $\mathrm{Sn} 3^{\mathrm{vii}}-\mathrm{Pd} 5-\mathrm{Sn} 4^{\mathrm{xii}}$ & $79.82(8)$ \\
\hline $\mathrm{Pd} 2-\mathrm{Pd} 5-\mathrm{Sn} 4^{\mathrm{xii}}$ & $121.13(7)$ \\
\hline $\mathrm{Pd} 4^{\mathrm{xiv}}-\mathrm{Pd} 5-\mathrm{Sn} 4^{\mathrm{xii}}$ & $58.75(6)$ \\
\hline $\mathrm{Pd} 7^{\mathrm{vii}}-\mathrm{Pd} 5-\mathrm{Sn} 4^{\mathrm{xii}}$ & $126.67(13)$ \\
\hline $\mathrm{Sn} 2-\mathrm{Pd} 5-\mathrm{Pd} 6^{\mathrm{xii}}$ & $123.11(9)$ \\
\hline $\mathrm{Sn} 5^{\mathrm{xii}}-\mathrm{Pd} 5-\mathrm{Pd} 6^{\mathrm{xii}}$ & $57.03(7)$ \\
\hline $\mathrm{Sn} 3^{\mathrm{ix}}-\mathrm{Pd} 5-\mathrm{Pd} 6^{\mathrm{xii}}$ & $119.99(5)$ \\
\hline
\end{tabular}

\begin{tabular}{|c|c|}
\hline $\mathrm{Pd} 4^{\mathrm{ii}}-\mathrm{Sn} 3-\mathrm{Pd} 5^{\mathrm{xvi}}$ & $144.37(7)$ \\
\hline $\mathrm{Pd} 7-\mathrm{Sn} 3-\mathrm{Pd} 5^{\mathrm{xvi}}$ & $62.65(6)$ \\
\hline $\mathrm{Pd} 5^{\mathrm{ix}}-\mathrm{Sn} 3-\mathrm{Pd} 5^{\mathrm{xvi}}$ & $103.75(3)$ \\
\hline $\mathrm{Pd} 8-\mathrm{Sn} 3-\mathrm{Pd} 6^{\mathrm{ii}}$ & $87.24(6)$ \\
\hline $\mathrm{Pd} 2^{\mathrm{xvi}}-\mathrm{Sn} 3-\mathrm{Pd} 6^{\mathrm{ii}}$ & $64.69(5)$ \\
\hline $\mathrm{Pd} 4^{\mathrm{ii}}-\mathrm{Sn} 3-\mathrm{Pd} 6^{\mathrm{ii}}$ & $61.01(6)$ \\
\hline $\mathrm{Pd} 7-\mathrm{Sn} 3-\mathrm{Pd} 6^{\mathrm{ii}}$ & $91.36(5)$ \\
\hline $\mathrm{Pd} 5^{\mathrm{ix}}-\mathrm{Sn} 3-\mathrm{Pd} 6^{\mathrm{ii}}$ & $123.02(9)$ \\
\hline $\mathrm{Pd} 5^{\mathrm{xvi}}-\mathrm{Sn} 3-\mathrm{Pd} 6^{\mathrm{ii}}$ & $126.05(9)$ \\
\hline $\mathrm{Pd} 8-\mathrm{Sn} 3-\mathrm{Pd} 6^{\mathrm{ix}}$ & $80.82(6)$ \\
\hline $\mathrm{Pd} 2^{\mathrm{xvi}}-\mathrm{Sn} 3-\mathrm{Pd} 6^{\mathrm{ix}}$ & $122.33(9)$ \\
\hline $\mathrm{Pd} 4^{\mathrm{ii}}-\mathrm{Sn} 3-\mathrm{Pd} 6^{\mathrm{ix}}$ & $123.59(10)$ \\
\hline $\mathrm{Pd} 7-\mathrm{Sn} 3-\mathrm{Pd} 6^{\mathrm{ix}}$ & $78.84(6)$ \\
\hline $\mathrm{Pd} 5^{\mathrm{ix}}-\mathrm{Sn} 3-\mathrm{Pd} 6^{\mathrm{ix}}$ & $62.38(7)$ \\
\hline $\mathrm{Pd} 5^{\mathrm{xvi}}-\mathrm{Sn} 3-\mathrm{Pd} 6^{\mathrm{ix}}$ & $61.68(7)$ \\
\hline $\mathrm{Pd} 6^{\mathrm{ii}}-\mathrm{Sn} 3-\mathrm{Pd} 6^{\mathrm{ix}}$ & $162.73(5)$ \\
\hline $\mathrm{Pd} 8-\mathrm{Sn} 3-\mathrm{Pd} 4$ & $56.50(5)$ \\
\hline $\mathrm{Pd} 22^{\mathrm{xvi}}-\mathrm{Sn} 3-\mathrm{Pd} 4$ & $94.42(7)$ \\
\hline $\mathrm{Pd} 44^{\mathrm{ii}}-\mathrm{Sn} 3-\mathrm{Pd} 4$ & $95.55(4)$ \\
\hline $\mathrm{Pd} 7-\mathrm{Sn} 3-\mathrm{Pd} 4$ & $55.90(4)$ \\
\hline $\mathrm{Pd} 5^{\mathrm{ix}}-\mathrm{Sn} 3-\mathrm{Pd} 4$ & $120.95(7)$ \\
\hline $\mathrm{Pd} 55^{\mathrm{xvi}}-\mathrm{Sn} 3-\mathrm{Pd} 4$ & $118.51(7)$ \\
\hline $\mathrm{Pd} 6{ }^{\mathrm{ii}}-\mathrm{Sn} 3-\mathrm{Pd} 4$ & $59.95(5)$ \\
\hline $\mathrm{Pd} 6^{\mathrm{ix}}-\mathrm{Sn} 3-\mathrm{Pd} 4$ & $102.87(5)$ \\
\hline $\mathrm{Pd} 6-\mathrm{Sn} 4-\mathrm{Pd} 7^{\mathrm{vii}}$ & $119.05(5)$ \\
\hline $\mathrm{Pd} 6-\mathrm{Sn} 4-\mathrm{Pd} 8^{\mathrm{xviii}}$ & $120.96(5)$ \\
\hline $\mathrm{Pd} 7^{\mathrm{vii}}-\mathrm{Sn} 4-\mathrm{Pd} 8^{\mathrm{xviii}}$ & $118.82(5)$ \\
\hline $\mathrm{Pd} 6-\mathrm{Sn} 4-\mathrm{Pd} 4$ viii & $66.95(7)$ \\
\hline $\mathrm{Pd} 7^{\mathrm{vii}}-\mathrm{Sn} 4-\mathrm{Pd} 4^{\mathrm{viii}}$ & $155.61(4)$ \\
\hline $\mathrm{Pd} 8^{\mathrm{xviii}}-\mathrm{Sn} 4-\mathrm{Pd} 4^{\mathrm{viii}}$ & $65.64(8)$ \\
\hline $\mathrm{Pd} 6-\mathrm{Sn} 4-\mathrm{Pd} 2$ & $65.30(6)$ \\
\hline $\mathrm{Pd} 7^{\mathrm{vii}}-\mathrm{Sn} 4-\mathrm{Pd} 2$ & $66.04(5)$ \\
\hline 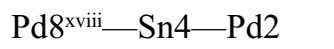 & $155.06(4)$ \\
\hline $\mathrm{Pd} 4^{\text {viii }}-\mathrm{Sn} 4-\mathrm{Pd} 2$ & $99.92(6)$ \\
\hline $\mathrm{Pd} 6-\mathrm{Sn} 4-\mathrm{Pd} 7^{\mathrm{viii}}$ & $92.76(6)$ \\
\hline $\mathrm{Pd} 7^{\mathrm{vii}}-\mathrm{Sn} 4-\mathrm{Pd} 7^{\mathrm{viii}}$ & $96.47(4)$ \\
\hline $\mathrm{Pd} 8^{\mathrm{xviii}}-\mathrm{Sn} 4-\mathrm{Pd} 7^{\mathrm{viii}}$ & $91.64(5)$ \\
\hline 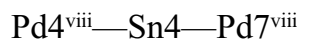 & $59.15(4)$ \\
\hline $\mathrm{Pd} 2-\mathrm{Sn} 4-\mathrm{Pd} 7^{\text {viii }}$ & $63.47(5)$ \\
\hline $\mathrm{Pd} 6-\mathrm{Sn} 4-\mathrm{Pd} 1^{\mathrm{xviii}}$ & $156.15(5)$ \\
\hline $\mathrm{Pd} 7^{\mathrm{vii}}-\mathrm{Sn} 4-\mathrm{Pd} 1^{\mathrm{xviii}}$ & $65.95(6)$ \\
\hline $\mathrm{Pd} 8^{\mathrm{xviii}}-\mathrm{Sn} 4-\mathrm{Pd} 1^{\mathrm{xviii}}$ & $64.49(7)$ \\
\hline $\mathrm{Pd} 4^{\mathrm{viii}}-\mathrm{Sn} 4-\mathrm{Pd} 1^{\mathrm{xviii}}$ & $99.16(4)$ \\
\hline $\mathrm{Pd} 2-\mathrm{Sn} 4-\mathrm{Pd} 1^{\mathrm{xviii}}$ & $100.19(5)$ \\
\hline $\mathrm{Pd} 7^{\mathrm{viii}}-\mathrm{Sn} 4-\mathrm{Pd} 1^{\mathrm{xviii}}$ & $63.42(6)$ \\
\hline $\mathrm{Pd} 6-\mathrm{Sn} 4-\mathrm{Pd} 5^{\mathrm{xv}}$ & $63.17(8)$ \\
\hline 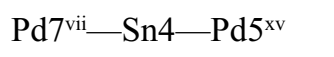 & $144.54(4)$ \\
\hline $\mathrm{Pd} 8^{\mathrm{xviii}}-\mathrm{Sn} 4-\mathrm{Pd} 5^{\mathrm{xv}}$ & $63.56(8)$ \\
\hline
\end{tabular}




\begin{tabular}{|c|c|c|c|}
\hline $\mathrm{Sn} 3^{\mathrm{vii}}-\mathrm{Pd} 5-\mathrm{Pd} 6^{\mathrm{xii}}$ & $60.54(8)$ & $\mathrm{Pd} 4^{\mathrm{viii}}-\mathrm{Sn} 4-\mathrm{Pd} 5^{\mathrm{xv}}$ & $59.85(4)$ \\
\hline $\mathrm{Pd} 2-\mathrm{Pd} 5-\mathrm{Pd} 6^{\mathrm{xii}}$ & $117.52(11)$ & $\mathrm{Pd} 2-\mathrm{Sn} 4-\mathrm{Pd} 5^{\mathrm{xv}}$ & $128.46(9)$ \\
\hline $\mathrm{Pd} 4^{\mathrm{xiv}}-\mathrm{Pd} 5-\mathrm{Pd} 6^{\mathrm{xii}}$ & $63.11(9)$ & $\mathrm{Pd} 7^{\mathrm{viii}}-\mathrm{Sn} 4-\mathrm{Pd} 5^{\mathrm{xv}}$ & $119.00(5)$ \\
\hline $\mathrm{Pd} 7^{\mathrm{vii}}-\mathrm{Pd} 5-\mathrm{Pd} 6^{\mathrm{xii}}$ & $76.17(9)$ & $\mathrm{Pd} 1^{\mathrm{xvii}}-\mathrm{Sn} 4-\mathrm{Pd} 5^{\mathrm{xv}}$ & $128.04(10)$ \\
\hline $\mathrm{Sn} 4^{\mathrm{xii}}-\mathrm{Pd} 5-\mathrm{Pd} 6^{\mathrm{xii}}$ & $54.07(6)$ & $\mathrm{Pd} 6-\mathrm{Sn} 4-\mathrm{Pd} 3^{\mathrm{xviii}}$ & $146.14(3)$ \\
\hline $\mathrm{Sn} 2-\mathrm{Pd} 5-\mathrm{Pd} 6$ & $56.88(7)$ & 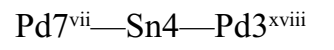 & $61.52(9)$ \\
\hline $\mathrm{Sn} 5^{\mathrm{xii}}-\mathrm{Pd} 5-\mathrm{Pd} 6$ & $122.99(9)$ & $\mathrm{Pd} 8^{\mathrm{xviii}}-\mathrm{Sn} 4-\mathrm{Pd} 3^{\text {xviii }}$ & $62.67(9)$ \\
\hline $\mathrm{Sn} 3^{3 \mathrm{x}}-\mathrm{Pd} 5-\mathrm{Pd} 6$ & $60.43(8)$ & $\mathrm{Pd} 4^{\text {viii }}-\mathrm{Sn} 4-\mathrm{Pd} 3^{\mathrm{xviii}}$ & $128.30(12)$ \\
\hline $\mathrm{Sn} 3^{\mathrm{vii}-\mathrm{Pd} 5-\mathrm{Pd} 6}$ & $119.04(5)$ & $\mathrm{Pd} 2-\mathrm{Sn} 4-\mathrm{Pd} 3^{\mathrm{xviii}}$ & $127.55(9)$ \\
\hline $\mathrm{Pd} 2-\mathrm{Pd} 5-\mathrm{Pd} 6$ & $62.01(5)$ & 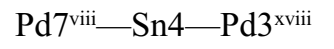 & $121.10(6)$ \\
\hline $\mathrm{Pd} 4^{\mathrm{xiv}}-\mathrm{Pd} 5-\mathrm{Pd} 6$ & $117.36(14)$ & $P d 1^{x v i i i}-S n 4-P d 3^{x v i i i}$ & $57.70(4)$ \\
\hline $\mathrm{Pd} 7^{\mathrm{vii}}-\mathrm{Pd} 5-\mathrm{Pd} 6$ & $103.40(5)$ & $\mathrm{Pd} 5^{\mathrm{xv}}-\mathrm{Sn} 4-\mathrm{Pd} 3^{\mathrm{xviii}}$ & $96.58(4)$ \\
\hline $\mathrm{Sn} 4^{\mathrm{xii}-\mathrm{Pd} 5-\mathrm{Pd} 6}$ & $126.25(10)$ & $\mathrm{Pd} 6-\mathrm{Sn} 4-\mathrm{Pd} 5$ & $62.73(8)$ \\
\hline$P d 6^{\text {xii }-P d 5-P d 6 ~}$ & $179.50(7)$ & $\mathrm{Pd} 77^{\mathrm{vii}}-\mathrm{Sn} 4-\mathrm{Pd} 5$ & $61.39(8)$ \\
\hline $\mathrm{Sn} 2-\mathrm{Pd} 5-\mathrm{Pd} 8^{\mathrm{ix}}$ & $59.68(6)$ & $\mathrm{Pd} 8^{\mathrm{xviii}}-\mathrm{Sn} 4-\mathrm{Pd} 5$ & $146.72(4)$ \\
\hline $\mathrm{Sn} 5^{\mathrm{xii}}-\mathrm{Pd} 5-\mathrm{Pd} 8^{\mathrm{ix}}$ & $119.04(5)$ & $\mathrm{Pd} 44^{\mathrm{viii}}-\mathrm{Sn} 4-\mathrm{Pd} 5$ & $129.67(10)$ \\
\hline $\mathrm{Sn} 3^{\mathrm{ix}}-\mathrm{Pd} 5-\mathrm{Pd} 8^{\mathrm{ix}}$ & $56.50(7)$ & $\mathrm{Pd} 2-\mathrm{Sn} 4-\mathrm{Pd} 5$ & $58.18(3)$ \\
\hline $\mathrm{Sn} 3^{\mathrm{vii}}-\mathrm{Pd} 5-\mathrm{Pd} 8^{\mathrm{ix}}$ & $123.70(7)$ & $\mathrm{Pd} 7^{\mathrm{viii}}-\mathrm{Sn} 4-\mathrm{Pd} 5$ & $121.64(6)$ \\
\hline $\mathrm{Pd} 2-\mathrm{Pd} 5-\mathrm{Pd} 8^{\mathrm{ix}}$ & $117.48(10)$ & $\mathrm{Pd} 1^{\mathrm{xviii}}-\mathrm{Sn} 4-\mathrm{Pd} 5$ & $127.34(9)$ \\
\hline $\mathrm{Pd} 4^{\mathrm{xiv}}-\mathrm{Pd} 5-\mathrm{Pd} 8^{\mathrm{ix}}$ & $61.79(6)$ & $\mathrm{Pd} 5^{\mathrm{xv}}-\mathrm{Sn} 4-\mathrm{Pd} 5$ & $96.82(3)$ \\
\hline $\mathrm{Pd} 7^{\mathrm{vii}}-\mathrm{Pd} 5-\mathrm{Pd} 8^{\mathrm{ix}}$ & $178.43(9)$ & $\mathrm{Pd} 3^{\text {xviii }}-\mathrm{Sn} 4-\mathrm{Pd} 5$ & $95.86(4)$ \\
\hline $\mathrm{Sn} 4^{\mathrm{xii}}-\mathrm{Pd} 5-\mathrm{Pd} 8^{\mathrm{ix}}$ & $54.16(5)$ & $\mathrm{Pd} 6-\mathrm{Sn} 5-\mathrm{Pd} 1^{\mathrm{xvii}}$ & $148.90(6)$ \\
\hline $\mathrm{Pd} 6^{\mathrm{xii}}-\mathrm{Pd} 5-\mathrm{Pd} 8^{\mathrm{ix}}$ & $104.12(5)$ & $\mathrm{Pd} 6-\mathrm{Sn} 5-\mathrm{Pd} 4^{\mathrm{vii}}$ & $67.13(9)$ \\
\hline $\mathrm{Pd} 6-\mathrm{Pd} 5-\mathrm{Pd} 8^{\mathrm{ix}}$ & $76.31(9)$ & $\mathrm{Pd} 1^{\mathrm{xvii}}-\mathrm{Sn} 5-\mathrm{Pd} 4^{\mathrm{viii}}$ & $108.52(4)$ \\
\hline $\mathrm{Sn} 2-\mathrm{Pd} 5-\mathrm{Sn} 4$ & $100.70(8)$ & $\mathrm{Pd} 6-\mathrm{Sn} 5-\mathrm{Pd} 7$ & $100.78(4)$ \\
\hline $\mathrm{Sn} 5^{\mathrm{xii}}-\mathrm{Pd} 5-\mathrm{Sn} 4$ & $79.87(5)$ & $\mathrm{Pd} 1^{\mathrm{xvii}}-\mathrm{Sn} 5-\mathrm{Pd} 7$ & $66.73(10)$ \\
\hline $\mathrm{Sn} 3^{3 \mathrm{x}}-\mathrm{Pd} 5-\mathrm{Sn} 4$ & $78.91(8)$ & $\mathrm{Pd} 44^{\mathrm{viii}}-\mathrm{Sn} 5-\mathrm{Pd} 7$ & $149.46(6)$ \\
\hline $\mathrm{Sn} 3^{\mathrm{vii}}-\mathrm{Pd} 5-\mathrm{Sn} 4$ & $100.63(6)$ & $\mathrm{Pd} 6-\mathrm{Sn} 5-\mathrm{Pd} 5^{\mathrm{xv}}$ & $64.05(8)$ \\
\hline $\mathrm{Pd} 2-\mathrm{Pd} 5-\mathrm{Sn} 4$ & $58.94(5)$ & $\mathrm{Pd} 1^{\mathrm{xvii}}-\mathrm{Sn} 5-\mathrm{Pd} 5^{\mathrm{xv}}$ & $143.48(12)$ \\
\hline $\mathrm{Pd} 4^{\mathrm{xiv}}-\mathrm{Pd} 5-\mathrm{Sn} 4$ & $121.17(9)$ & $\mathrm{Pd} 4^{\mathrm{viii}}-\mathrm{Sn} 5-\mathrm{Pd} 5^{\mathrm{xv}}$ & $62.38(5)$ \\
\hline $\mathrm{Pd} 7^{\mathrm{vii}}-\mathrm{Pd} 5-\mathrm{Sn} 4$ & $53.93(4)$ & $\mathrm{Pd} 7-\mathrm{Sn} 5-\mathrm{Pd} 5^{\mathrm{xv}}$ & $139.46(6)$ \\
\hline 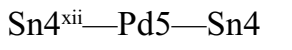 & $179.39(14)$ & $\mathrm{Pd} 6-\mathrm{Sn} 5-\mathrm{Pd} 8^{\text {viii }}$ & $87.99(6)$ \\
\hline $\mathrm{Pd} 6^{\mathrm{xii}}-\mathrm{Pd} 5-\mathrm{Sn} 4$ & $126.50(10)$ & $\mathrm{Pd} 1^{\mathrm{xvii}}-\mathrm{Sn} 5-\mathrm{Pd} 8^{\mathrm{viii}}$ & $64.94(5)$ \\
\hline $\mathrm{Pd} 6-\mathrm{Pd} 5-\mathrm{Sn} 4$ & $53.18(6)$ & $\mathrm{Pd} 4^{\text {viii }}-\mathrm{Sn} 5-\mathrm{Pd} 8^{\text {viii }}$ & $61.40(4)$ \\
\hline $\mathrm{Pd} 8^{\mathrm{ix}}-\mathrm{Pd} 5-\mathrm{Sn} 4$ & $125.24(13)$ & $\mathrm{Pd} 7-\mathrm{Sn} 5-\mathrm{Pd} 8^{\text {viii }}$ & $91.30(5)$ \\
\hline $\mathrm{Sn} 4-\mathrm{Pd} 6-\mathrm{Sn} 2$ & $110.61(9)$ & $\mathrm{Pd} 5^{\mathrm{xv}}-\mathrm{Sn} 5-\mathrm{Pd} 8^{\mathrm{viii}}$ & $123.30(7)$ \\
\hline $\mathrm{Sn} 4-\mathrm{Pd} 6-\mathrm{Sn} 5$ & $109.00(8)$ & $\mathrm{Pd} 6-\mathrm{Sn} 5-\mathrm{Pd} 3^{\mathrm{xvii}}$ & $140.17(7)$ \\
\hline $\mathrm{Sn} 2-\mathrm{Pd} 6-\mathrm{Sn} 5$ & $139.69(6)$ & $\mathrm{Pd} 1^{\mathrm{xvii}}-\mathrm{Sn} 5-\mathrm{Pd} 3^{\mathrm{xvii}}$ & $61.14(6)$ \\
\hline $\mathrm{Sn} 4-\mathrm{Pd} 6-\mathrm{Pd} 9$ & $154.46(4)$ & $\mathrm{Pd} 4^{\mathrm{viii}}-\mathrm{Sn} 5-\mathrm{Pd} 3^{\mathrm{xvii}}$ & $144.02(11)$ \\
\hline $\mathrm{Sn} 2-\mathrm{Pd} 6-\mathrm{Pd} 9$ & $73.65(6)$ & $\mathrm{Pd} 7-\mathrm{Sn} 5-\mathrm{Pd} 3^{\mathrm{xvii}}$ & $62.41(6)$ \\
\hline $\mathrm{Sn} 5-\mathrm{Pd} 6-\mathrm{Pd} 9$ & $73.96(6)$ & $\mathrm{Pd} 5^{\mathrm{xv}}-\mathrm{Sn} 5-\mathrm{Pd} 3^{\mathrm{xvii}}$ & $104.05(4)$ \\
\hline $\mathrm{Sn} 4-\mathrm{Pd} 6-\mathrm{Pd} 4$ & $146.07(6)$ & $\mathrm{Pd} 8^{\mathrm{viii}}-\mathrm{Sn} 5-\mathrm{Pd} 3^{\mathrm{xvii}}$ & $125.70(8)$ \\
\hline $\mathrm{Sn} 2-\mathrm{Pd} 6-\mathrm{Pd} 4$ & $73.51(6)$ & $\mathrm{Pd} 6-\mathrm{Sn} 5-\mathrm{Pd} 7^{\mathrm{ix}}$ & $80.39(6)$ \\
\hline $\mathrm{Sn} 5-\mathrm{Pd} 6-\mathrm{Pd} 4$ & $69.63(6)$ & $\mathrm{Pd} 1^{\mathrm{xvii}}-\mathrm{Sn} 5-\mathrm{Pd} 7^{\mathrm{ix}}$ & $121.86(8)$ \\
\hline $\mathrm{Pd} 9-\mathrm{Pd} 6-\mathrm{Pd} 4$ & $59.38(5)$ & $\mathrm{Pd} 4^{\mathrm{viii}}-\mathrm{Sn} 5-\mathrm{Pd} 7^{\mathrm{ix}}$ & $123.48(8)$ \\
\hline $\mathrm{Sn} 4-\mathrm{Pd} 6-\mathrm{Sn} 3^{\text {viii }}$ & $88.76(5)$ & $\mathrm{Pd} 7-\mathrm{Sn} 5-\mathrm{Pd} 7^{\mathrm{ix}}$ & $78.91(5)$ \\
\hline $\mathrm{Sn} 2-\mathrm{Pd} 6-\mathrm{Sn} 3^{\text {viii }}$ & $81.94(5)$ & $\mathrm{Pd} 5^{\mathrm{xv}}-\mathrm{Sn} 5-\mathrm{Pd} 7^{\mathrm{ix}}$ & $62.05(6)$ \\
\hline $\mathrm{Sn} 5-\mathrm{Pd} 6-\mathrm{Sn} 3^{\mathrm{viii}}$ & $91.82(6)$ & $\mathrm{Pd} 8^{\mathrm{viii}}-\mathrm{Sn} 5-\mathrm{Pd} 7^{\mathrm{ix}}$ & $163.04(5)$ \\
\hline
\end{tabular}




\begin{tabular}{|c|c|c|c|}
\hline $\mathrm{Pd} 9-\mathrm{Pd} 6-\mathrm{Sn} 3^{\text {viii }}$ & $116.71(6)$ & $\mathrm{Pd} 3^{\mathrm{xvii}}-\mathrm{Sn} 5-\mathrm{Pd} 7^{\mathrm{ix}}$ & $61.63(7)$ \\
\hline $\mathrm{Pd} 4-\mathrm{Pd} 6-\mathrm{Sn} 3^{\text {viii }}$ & $57.88(6)$ & $\mathrm{Pd} 6-\mathrm{Sn} 5-\mathrm{Pd} 4$ & $56.77(6)$ \\
\hline $\mathrm{Sn} 4-\mathrm{Pd} 6-\mathrm{Sn} 3^{\mathrm{ix}}$ & $82.84(4)$ & $\mathrm{Pd} 1^{\mathrm{xvii}}-\mathrm{Sn} 5-\mathrm{Pd} 4$ & $94.62(5)$ \\
\hline $\mathrm{Sn} 2-\mathrm{Pd} 6-\mathrm{Sn} 3^{\mathrm{ix}}$ & $94.90(8)$ & $\mathrm{Pd} 4^{\mathrm{vii}}-\mathrm{Sn} 5-\mathrm{Pd} 4$ & $95.82(4)$ \\
\hline Sn5-Pd6-Sn $3^{\text {ix }}$ & $97.05(8)$ & $\mathrm{Pd} 7-\mathrm{Sn} 5-\mathrm{Pd} 4$ & $56.10(7)$ \\
\hline $\mathrm{Pd} 9-\mathrm{Pd} 6-\mathrm{Sn} 3^{\mathrm{ix}}$ & $71.64(3)$ & $\mathrm{Pd} 5^{\mathrm{xv}}-\mathrm{Sn} 5-\mathrm{Pd} 4$ & $120.73(11)$ \\
\hline $\mathrm{Pd} 4-\mathrm{Pd} 6-\mathrm{Sn} 3^{\mathrm{ix}}$ & $131.01(6)$ & $\mathrm{Pd} 8^{\mathrm{viii}}-\mathrm{Sn} 5-\mathrm{Pd} 4$ & $59.62(4)$ \\
\hline $\mathrm{Sn} 3^{\text {viii }}-\mathrm{Pd} 6-\mathrm{Sn} 3^{\mathrm{ix}}$ & $169.38(7)$ & $\mathrm{Pd} 3^{\mathrm{xvii}}-\mathrm{Sn} 5-\mathrm{Pd} 4$ & $118.48(10)$ \\
\hline $\mathrm{Sn} 4-\mathrm{Pd} 6-\mathrm{Pd} 5^{\mathrm{xv}}$ & $62.76(5)$ & $\mathrm{Pd} 7^{\mathrm{ix}}-\mathrm{Sn} 5-\mathrm{Pd} 4$ & $103.49(4)$ \\
\hline
\end{tabular}

Symmetry codes: (i) $x-1, y-1, z$; (ii) $x-y,-y+1,-z+1 / 3$; (iii) $x-y,-y,-z+1 / 3$; (iv) $-x+y,-x, z+1 / 3$; (v) $x-y-1,-y,-z+1 / 3$; (vi) $x-1, y, z$; (vii) $x, y-1, z$; (viii) $x-y+1,-y+1,-z+1 / 3$; (ix) $y, x,-z$; (x) $y-1, x-1,-z$; (xi) $-y, x-y, z-1 / 3$; (xii) $y, x-1,-z$; (xiii) $-x+y+1,-x+1, z+1 / 3$; (xiv) $-y+1, x-y, z-1 / 3$; (xv) $y+1$, $x,-z$; (xvi) $x, y+1, z$; (xvii) $x+1, y+1, z$; (xviii) $x+1, y, z$. 